TRANSACTIONS OF THE

AMERICAN MATHEMATICAL SOCIETY

Volume 361, Number 6, June 2009, Pages 3159-3187

S 0002-9947(09)04669-8

Article electronically published on January 22, 2009

\title{
MULTIBUMP SOLUTIONS AND CRITICAL GROUPS
}

\author{
GIANNI ARIOLI, ANDRZEJ SZULKIN, AND WENMING ZOU
}

\begin{abstract}
We consider the Newtonian system $-\ddot{q}+B(t) q=W_{q}(q, t)$ with $B, W$ periodic in $t, B$ positive definite, and show that for each isolated homoclinic solution $q_{0}$ having a nontrivial critical group (in the sense of Morse theory), multibump solutions (with $2 \leq k \leq \infty$ bumps) can be constructed by gluing translates of $q_{0}$. Further we show that the collection of multibumps is semiconjugate to the Bernoulli shift. Next we consider the Schrödinger equation $-\Delta u+V(x) u=g(x, u)$ in $\mathbb{R}^{N}$, where $V, g$ are periodic in $x_{1}, \ldots, x_{N}$, $\sigma(-\Delta+V) \subset(0, \infty)$, and we show that similar results hold in this case as well. In particular, if $g(x, u)=|u|^{2^{*}-2} u, N \geq 4$ and $V$ changes sign, then there exists a solution minimizing the associated functional on the Nehari manifold. This solution gives rise to multibumps if it is isolated.
\end{abstract}

\section{INTRODUCTION AND STATEMENT OF MAIN RESULT}

In this paper we will be concerned with the existence of multibump solutions for Newtonian systems of ordinary differential equations and for semilinear partial differential equations of Schrödinger type. Consider first the Newtonian system

$$
-\ddot{q}+B(t) q=W_{q}(q, t), \quad q \in \mathbb{R}^{N}, t \in \mathbb{R},
$$

where $B$ and $W$ satisfy the following assumptions:

$\left(H_{1}\right) \quad B$ is an $N \times N$ symmetric positive definite matrix with continuous 1-periodic entries;

$\left(H_{2}\right) W \in C\left(\mathbb{R}^{N} \times \mathbb{R}, \mathbb{R}\right), W_{q} \in C\left(\mathbb{R}^{N} \times \mathbb{R}, \mathbb{R}^{N}\right), W_{q q} \in C\left(\mathbb{R}^{N} \times \mathbb{R}, \mathbb{R}^{N^{2}}\right)$ and $W$ is 1 -periodic in $t$;

$\left(H_{3}\right) W_{q}(0, t)=0$ and $W_{q q}(0, t)=0$ for all $t$.

Note that $q=0$ satisfies (1.1) according to $\left(H_{3}\right)$. A solution $q$ of (1.1) will be called homoclinic to 0 (or homoclinic for short) if $q \not \equiv 0$ and $q(t), \dot{q}(t) \rightarrow 0$ as $|t| \rightarrow \infty$.

Received by the editors July 12, 2007.

2000 Mathematics Subject Classification. Primary 37J45; Secondary 34C28, 35J20, 35Q55.

Key words and phrases. Multibump solution, critical group, Bernoulli shift, Newtonian system, Schrödinger equation, critical exponent.

The first author was supported in part by the MIUR project "Equazioni alle derivate parziali e disuguaglianze funzionali: aspetti quantitativi, proprietà geometriche e qualitative, applicazioni".

The second author was supported in part by the Swedish Research Council.

The third author was supported by NSFC (10571096 and 10871109), SRF-ROCS-SEM and the program of the Ministry of Education in China for New Century Excellent Talents in Universities of China.

(C)2009 American Mathematical Society 
Let $E:=H^{1}\left(\mathbb{R}, \mathbb{R}^{N}\right)$ be the usual Sobolev space and set

$$
\langle q, \tilde{q}\rangle:=\int_{\mathbb{R}}(\dot{q} \cdot \dot{\tilde{q}}+B(t) q \cdot \tilde{q}) d t, \quad\|q\|:=\langle q, q\rangle^{1 / 2} .
$$

Since $B$ is positive definite and 1-periodic, $\langle.,$.$\rangle is an equivalent inner product in$ $E$. It is well known that if

$$
\Phi(q):=\frac{1}{2}\|q\|^{2}-\int_{\mathbb{R}} W(q, t) d t,
$$

then $\Phi \in C^{2}(E, \mathbb{R})$ and critical points $q \neq 0$ of $\Phi$ correspond to homoclinic solutions of (1.1).

For a function $q: \mathbb{R} \rightarrow \mathbb{R}^{N}$ and $\theta \in \mathbb{R}$, let

$$
(\theta * q)(t):=q(t-\theta) .
$$

Recall that the $r$-th critical group of $\Phi$ at an isolated critical point $q_{0}$ is defined by

$$
C_{r}\left(\Phi, q_{0}\right)=H_{r}\left(\Phi^{c} \cap U, \Phi^{c} \cap U \backslash\left\{q_{0}\right\}\right),
$$

where $c=\Phi\left(q_{0}\right), \Phi^{c}=\{q \in E: \Phi(q) \leq c\}, U$ is a neighborhood of $q_{0}$ and $H_{r}$ is the $r$-th singular homology group with coefficients in some field $\mathcal{F}$ (cf. [10, 26]). We shall write $C_{*}\left(\Phi, q_{0}\right)=\left\{C_{r}\left(\Phi, q_{0}\right): r \in \mathbb{N} \cup\{0\}\right\}$. Our main result on (1.1) which we formulate below asserts that if $q_{0}$ is an isolated homoclinic solution with $C_{*}\left(\Phi, q_{0}\right) \neq 0$, then there must necessarily exist infinitely many homoclinics which can be obtained by putting together translates of $q_{0}$ and adding a small correction term. Note that this result does not apply to autonomous systems because if $q_{0}$ is a homoclinic, then so is $\theta * q_{0}$ for any $\theta \in \mathbb{R}$; hence $q_{0}$ can never be isolated.

Theorem 1.1. Suppose the hypotheses $\left(H_{1}\right)-\left(H_{3}\right)$ are satisfied and $q_{0}$ is an isolated homoclinic solution of (1.1) such that $C_{*}\left(\Phi, q_{0}\right) \neq 0$. For each $\delta>0$ there exists $a \in \mathbb{N}$ with the property that if $k \geq 2, \theta_{1}, \theta_{2}, \ldots, \theta_{k} \in \mathbb{Z}$ and $\theta_{i+1}-\theta_{i} \geq a$ for all $i=$ $1,2, \ldots, k-1$, then there is $v \in E$ such that $\|v\|_{\infty} \leq \delta$ and $\bar{q}=\theta_{1} * q_{0}+\cdots+\theta_{k} * q_{0}+v$ is a homoclinic solution of (1.1).

Since $\theta_{1} * q_{0}, \ldots, \theta_{k} * q_{0}$ are translates of $q_{0}$ and $v$ is small, $\bar{q}$ is called a $k$-bump solution. As $\theta_{i+1}-\theta_{i}$ may be chosen arbitrarily large, for each $k$ there are infinitely many $k$-bump solutions. We emphasize that the minimal distance $a$ between the centers of two consecutive bumps is independent of the choice of $k$.

The first paper where modern global variational methods have been employed in order to find homoclinic solutions for a Hamiltonian system seems to be 12 . The Hamiltonian considered there was of the form $H(z, t)=\frac{1}{2} A z \cdot z+G(z, t)$, with $G$ periodic in $t$, strictly convex in $z$ and $G_{z}$ superlinear. Subsequently multibump solutions for this system have been found in 29, 30. In particular, in 30 the minimal distance between the bumps has been made independent of their number; moreover, it has been shown that there are (nonhomoclinic) solutions with infinitely many bumps and there is an almost continuous embedding of the Bernoulli shift on two symbols into the set of all solutions. Existence of multibump solutions for (1.1) (with $a$ possibly depending on $k$ ) has been shown in [13, under a superlinearity condition on $W_{q}$, and in [15 periodic $k$-bump solutions as well as (nonperiodic) solutions with infinitely many bumps have been shown to exist. In these papers the multibumps have been obtained starting from a mountain pass point at a level $c$, under the assumption that there are only finitely many geometrically distinct homoclinics below a somewhat higher level $c+\varepsilon$. In Theorem 1.1 we show that a 
multibump construction can be carried out if $q_{0}$ is any isolated homoclinic having a nontrivial critical group.

A particular case of the Schrödinger equation we consider is

$$
-\Delta u+V(x) u=|u|^{2^{*}-2} u, \quad x \in \mathbb{R}^{N}, u \in \mathbb{R},
$$

where $2^{*}$ is the critical Sobolev exponent, $V$ is 1 -periodic in $x_{1}, \ldots, x_{N}$ and the spectrum $\sigma(-\Delta+V) \subset(0, \infty)$. We show that if $V$ changes sign and $N \geq 4$, then (1.4) has a solution $u \neq 0$ which is a minimizer for the associated functional on the Nehari manifold. Moreover, there exist multibumps whenever this solution is isolated. We emphasize that our result implies (1.4) always has infinitely many solutions which are geometrically distinct in the sense that they are not translates of each other by elements of $\mathbb{Z}^{N}$.

There is an extensive literature on multibump solutions, both for ordinary and partial differential equations. In addition to the references given above, see e.g. 1, 2, 3, 14, 23, 24, 25, 34. (a much more comprehensive bibliography may be found in [28). Note that Theorem 3.4 in 1 resembles our Theorems 1.1 and 6.1 if $u_{0}$ is an isolated solution having nontrivial local degree, then there exist multibumps which can be obtained by gluing translates of $u_{0}$. However, $a$ may depend on $k$ in [1]. We also mention the paper [5] where multibump solutions have been found for an infinite lattice of particles (a Fermi-Pasta-Ulam type problem).

This paper is organized as follows. In Section 2 we prove, by a relatively simple argument, Theorem 1.1. Section 3 discusses some consequences of the results obtained and the arguments used so far. It is shown that different homoclinics can be glued into multibumps and that there exist multibump periodic solutions as well as nonperiodic solutions with infinitely many bumps. This should be compared with the results in 13, 15. In Section 4 we show that a dynamical system related to (1.1) is semiconjugate to the Bernoulli shift (a somewhat different viewpoint has been taken in 230; see Remark 4.2). In Section 5 a homoclinic $q_{0}$ with $C_{1}\left(\Phi, q_{0}\right) \neq 0$ is shown to exist under suitable assumptions, either by finding a mountain pass point or by minimizing $\Phi$ over the Nehari manifold. It is also shown that for a special case of (1.1) (a nonautonomous perturbation of an autonomous system) there exists another homoclinic $\tilde{q}$ such that $C_{2}(\Phi, \tilde{q}) \neq 0$. This $\tilde{q}$ is a mountain pass point for the restriction of $\Phi$ to the Nehari manifold and a kind of linking point for the functional on the full space. In Section 6 the results on (1.1) are extended to Schrödinger equations. In particular, (1.4) is considered.

After this paper was already written, the authors learned about the recent thesis of $\mathrm{Xu}$ [37. One of the main results there extends earlier work by Y.Y. Li and asserts that the equation

$$
-\Delta u=K(x) u^{2^{*}-1}, \quad x \in \mathbb{R}^{N}, u \in \mathbb{R},
$$

where $K$ is periodic in $x_{1}, \ldots, x_{N}$ and satisfies some additional hypotheses, possesses positive $k$-bump solutions for each finite $k$. Since both the results and the methods of [37] are very different from ours, our paper remains unchanged.

Notation. $\|\cdot\|_{p}$ is the usual $L^{p}\left(\mathbb{R}^{N}\right)$-norm, $1 \leq p \leq \infty, \rightarrow$ denotes weak convergence, $\cdot$ is the standard inner product in $\mathbb{R}^{N}$ and $\langle.,$.$\rangle an inner product in an$ infinite-dimensional Hilbert space. For a functional $\Phi$ defined on a Hilbert space $E$ we identify (via duality) $\Phi^{\prime}(x) \in E^{*}$ with an element of $E$ and we regard $\Phi^{\prime \prime}(x)$ as a linear mapping from $E$ into itself. We also set

$$
\Phi^{c}:=\{x \in E: \Phi(x) \leq c\} \quad \text { and } \quad K(c):=\left\{x \in E: \Phi(x)=c, \Phi^{\prime}(x)=0\right\} .
$$




\section{Proof of Theorem 1.1}

We begin with the well-known Liapunov-Schmidt reduction in a neighborhood of the solution $q_{0}$. Let $L:=\Phi^{\prime \prime}\left(q_{0}\right)$. Then $q \in N(L)$ (the nullspace of $L$ ) if and only if $q \in E$ and

$$
-\ddot{q}+B(t) q=W_{q q}\left(q_{0}, t\right) q .
$$

Suppose $N(L) \neq\{0\}$ (the other case is simpler; see Remark 2.13), let $Q: E \rightarrow R(L)$ be the orthogonal projection $(R(L)$ denotes the range of $L)$ and write $q=q_{0}+n+v$, where $n \in N(L), v \in R(L)$. Since $Q \Phi^{\prime \prime}\left(q_{0}\right)=\left.L\right|_{R(L)}: R(L) \rightarrow R(L)$ is invertible, it follows from the implicit function theorem that there exists a neighborhood $U$ of 0 in $E$ and a $C^{1}$-function $v=v(n)$ such that for $n+v \in U$, we have

$$
Q \Phi^{\prime}\left(q_{0}+n+v\right)=0 \quad \text { if and only if } v=v(n) .
$$

Moreover, $v(0)=0$ and $v^{\prime}(0)=0$. Hence in a neighborhood of $q_{0}$,

$$
\Phi^{\prime}\left(q_{0}+n+v\right)=0 \quad \text { if and only if } \quad(I-Q) \Phi^{\prime}\left(q_{0}+n+v(n)\right)=0 .
$$

Let $\varphi(n):=\Phi\left(q_{0}+n+v(n)\right),\|n\| \leq \varepsilon$, where $\varepsilon>0$ is appropriately small. By (2.2) and since $v^{\prime}(n) \in R(L)$,

$$
\varphi^{\prime}(n)=\left\langle\Phi^{\prime}\left(q_{0}+n+v(n)\right), \cdot+v^{\prime}(n) \cdot\right\rangle=\left\langle(I-Q) \Phi^{\prime}\left(q_{0}+n+v(n)\right), \cdot\right\rangle,
$$

therefore restricting $\Phi$ to a small neighborhood of $q_{0}$, we infer that $\varphi^{\prime}(n)=0$ if and only if $q_{0}+n+v(n)$ is a critical point of $\Phi$. Moreover, since $q_{0}$ is isolated, we may assume that $\varphi$ has no other critical point than 0 . Since $\Phi$ satisfies the Palais-Smale condition in a neighborhood of $q_{0}$ as will be shown in a moment, $C_{*}\left(\Phi, q_{0}\right)=C_{*-M^{-}(L)}(\varphi, 0)$ according to the shifting theorem [10, Theorem I.5.4], [26. Theorem 8.4] $\left(M^{-}(L)\right.$ is the Morse index of $\left.L\right)$. Hence keeping in mind that $C_{*}\left(\Phi, q_{0}\right) \neq 0$, we obtain

Lemma 2.1. $C_{*}(\varphi, 0) \neq 0$.

The next conclusion is probably well known, but we could not find any convenient reference.

Lemma 2.2. If $U$ is a sufficiently small neighborhood of $q_{0}$, then $\Phi$ satisfies the Palais-Smale condition on $\bar{U}$.

Proof. For the purpose of this proof we may assume $q_{0}=0$ and $L=\Phi^{\prime \prime}(0)$. Suppose $\left(q_{m}\right) \subset \bar{U}, \Phi^{\prime}\left(q_{m}\right) \rightarrow 0$ and write $q_{m}=n_{m}+v_{m} \in N(L) \oplus R(L), \Phi^{\prime}\left(q_{m}\right)=$ $L v_{m}+M\left(q_{m}\right)$. Then $M\left(q_{m}\right)=\Phi^{\prime}\left(q_{m}\right)-L v_{m}$, so $M^{\prime}(0)=0$. Since

$$
L v_{m}+Q M\left(n_{m}+v_{m}\right)=: w_{m} \rightarrow 0
$$

and, according to (2.2),

$$
L v\left(n_{m}\right)+Q M\left(n_{m}+v\left(n_{m}\right)\right)=0,
$$

we have

$$
v_{m}-v\left(n_{m}\right)=L^{-1} w_{m}-L^{-1} Q\left(M\left(n_{m}+v_{m}\right)-M\left(n_{m}+v\left(n_{m}\right)\right)\right) .
$$

Shrinking $U$ if necessary and using $M^{\prime}(0)=0$ gives

$$
\left\|v_{m}-v\left(n_{m}\right)\right\| \leq\left\|L^{-1} w_{m}\right\|+\frac{1}{2}\left\|v_{m}-v\left(n_{m}\right)\right\| .
$$


Hence $v_{m}-v\left(n_{m}\right) \rightarrow 0$. Since $\operatorname{dim} N(L)<\infty$, we obtain after passing to a subsequence that $n_{m} \rightarrow \bar{n}, v\left(n_{m}\right) \rightarrow v(\bar{n})$ and $v_{m} \rightarrow v(\bar{n})$. (Since $q_{0}$ is an isolated critical point, it follows in fact that $n_{m} \rightarrow 0$ and $v_{m} \rightarrow 0$.)

Now let $\omega \in \mathcal{C}_{0}^{\infty}(\mathbb{R},[0,1])$ be a function such that $\omega(t)=1$ if $|t| \leq 1 / 8$ and $\omega(t)=0$ if $|t| \geq 1 / 4$. Set

$$
q^{a}(t):=\omega(t / a) q(t), \quad a>0,
$$

and note that $\operatorname{supp} q^{a} \subset[-a / 4, a / 4]$, so $\operatorname{supp}\left(\theta_{1} * q^{a}\right) \cap \operatorname{supp}\left(\theta_{2} * q^{a}\right)=\emptyset$ whenever $\theta_{2}-\theta_{1} \geq a$. We shall repeatedly use the following notation for $a>0, \theta_{0} \in \mathbb{Z}$ and a set $S \subset E$ :

$$
S^{a}:=\left\{q^{a}: q \in S\right\}, \theta_{0} * S:=\left\{\theta_{0} * q: q \in S\right\} \text { and } \theta_{0} * S^{a}:=\theta_{0} *\left(S^{a}\right) .
$$

It is easy to see that $q^{a} \rightarrow q$ in $E$ as $a \rightarrow \infty$, and if $S$ is a compact set, the convergence is uniform for $q \in S$. Hence it follows from (2.2) that

$$
Q \Phi^{\prime}\left(\left(q_{0}+n+v(n)\right)^{a}\right) \rightarrow 0 \text { uniformly in } n \text { (where }\|n\| \leq \varepsilon \text { ) as } a \rightarrow \infty .
$$

Since the remaining part of the proof of Theorem 1.1 is rather lengthy, we describe the main steps. We acknowledge that we have made crucial use of some ideas that appear in the paper [7] by Berti and Bolle. In order to get the number $k$ of bumps independent of $a$ we introduce a new norm in $E$ which is equivalent to the old one for each fixed $k$ but the equivalence is not uniform in $k$. Then in Lemmas 2.4 2.8 we obtain a number of $k$-independent auxiliary estimates which we employ together with the contraction mapping principle in order to find $w(\theta, z)$ $\left(\theta=\left(\theta_{1}, \ldots, \theta_{k}\right), z=\left(n_{1}, \ldots, n_{k}\right) \in N(L)^{k}\right)$ such that the critical points of

$$
\widetilde{\varphi}(z):=\Phi\left(\sum_{i=1}^{k} \theta_{i} *\left(q_{0}+n_{i}+v\left(n_{i}\right)\right)^{a}+w(\theta, z)\right)
$$

correspond to $k$-bump solutions of (1.1). This is done in Lemmas 2.9, 2.11 and Proposition 2.10 Finally we show using Künneth's formula and Lemma 2.1 that $\widetilde{\varphi}$ must necessarily have a critical point (Künneth's formula has also been used in [30] though in a rather different way).

Let $a, k \in \mathbb{N}, k \geq 2$ and

$$
\Theta_{k}^{a}:=\left\{\theta=\left(\theta_{1}, \ldots, \theta_{k}\right) \in \mathbb{Z}^{k}: \theta_{i+1}-\theta_{i} \geq a\right\} .
$$

For a given $\theta \in \Theta_{k}^{a}$, let

$$
I_{i}:=\left(\frac{1}{2}\left(\theta_{i-1}+\theta_{i}\right), \frac{1}{2}\left(\theta_{i}+\theta_{i+1}\right)\right), \quad 1 \leq i \leq k,
$$

where $\theta_{0}:=-\infty$ and $\theta_{k+1}:=+\infty$. Following [7, we introduce a new norm in $E$ by setting

$$
\|q\|_{\theta}:=\max _{1 \leq i \leq k}\|q\|_{i}, \text { where }\|q\|_{i}^{2}:=\int_{I_{i}}\left(|\dot{q}|^{2}+B(t) q \cdot q\right) d t .
$$

The space $E$ with this norm will be denoted by $E_{\theta}$ and the space of bounded linear operators on $E_{\theta}$ by $\mathcal{L}\left(E_{\theta}\right)$.

Lemma 2.3. $\|q\|_{\theta} \leq\|q\| \leq k^{1 / 2}\|q\|_{\theta}$ and $\|q\|_{\infty} \leq c\|q\|_{\theta}$ for some $c>0$ and all $a \in \mathbb{N}, k \geq 2, \theta \in \Theta_{k}^{a}, q \in E$. 
Proof. The inequality $\|q\|_{\theta} \leq\|q\| \leq k^{1 / 2}\|q\|_{\theta}$ is an immediate consequence of the definition of the $\theta$-norm, and $\|q\|_{\infty} \leq c\|q\|_{\theta}$ follows from the Sobolev embedding theorem applied on each $I_{i}$.

Lemma 2.4. Suppose $a \in \mathbb{N}, w \in E,\|w\|_{\theta}=\|w\|_{j}$ and $\chi_{j} \in C^{\infty}(\mathbb{R},[0,1])$ is a function such that $\chi_{j}(t)=1$ for all $t \in I_{j}, \chi_{j}(t)=0$ if $t \notin \widetilde{I}_{j}:=\{t \in \mathbb{R}$ : $\left.\operatorname{dist}\left(t, I_{j}\right)<a / 4\right\}$ (dist denotes the distance) and $\left|\dot{\chi}_{j}(t)\right| \leq 8 / a$ for all $t$. There exists $a_{0} \in \mathbb{N}$ such that if $a \geq a_{0}$, then $\frac{1}{2}\|w\|_{\theta}^{2} \leq\left\langle w, \chi_{j} w\right\rangle \leq 4\|w\|_{\theta}^{2}$ and $\left\|\chi_{j} w\right\| \leq$ $2\|w\|_{\theta}$.

Proof. For a large enough,

$$
\begin{aligned}
\left\langle w, \chi_{j} w\right\rangle & =\int_{\mathbb{R}}\left(\chi_{j}(t)\left(|\dot{w}|^{2}+B(t) w \cdot w\right)+\dot{\chi}_{j}(t) w \cdot \dot{w}\right) d t \\
& \geq\|w\|_{j}^{2}-\frac{c_{1}}{a}\|w\|_{H^{1}\left(I_{j-1} \cup I_{j} \cup I_{j+1}\right)}^{2} \geq\|w\|_{j}^{2}-\frac{3 c_{1}}{a}\|w\|_{j}^{2} \geq \frac{1}{2}\|w\|_{j}^{2}
\end{aligned}
$$

(if $j=1$ or $k$, then $I_{j-1}$ or $I_{j+1}$ is empty). Hence $\left\langle w, \chi_{j} w\right\rangle \geq \frac{1}{2}\|w\|_{\theta}^{2}$. Similarly,

$$
\left\langle w, \chi_{j} w\right\rangle \leq 3\|w\|_{j}^{2}+\frac{3 c_{2}}{a}\|w\|_{j}^{2} \leq 4\|w\|_{j}^{2}
$$

and

$$
\left\|\chi_{j} w\right\|^{2} \leq 3\|w\|_{j}^{2}+\frac{3 c_{3}}{a}\|w\|_{j}^{2} \leq 4\|w\|_{j}^{2}
$$

Let

$$
V_{a}:=\left(\bigoplus_{i=1}^{k} \theta_{i} * N(L)^{a}\right)^{\perp} \quad \text { and } \quad P_{a}: E \rightarrow V_{a},
$$

where $\perp$ denotes the orthogonal complement in $E$ and $P_{a}$ is the orthogonal projection on $V_{a}$. Notice that $\theta_{i} * N(L)^{a}$ has support in $I_{i}$.

Lemma 2.5. There exist $c>0, \delta>0$ and $a_{0} \in \mathbb{N}$ such that

$$
\left\|\Phi^{\prime \prime}\left(\sum_{i=1}^{k} \theta_{i} * q_{0}^{a}+q\right) w\right\|_{\theta} \geq c\|w\|_{\theta}
$$

for all $a \in \mathbb{N}, a \geq a_{0}, k \geq 2, \theta \in \Theta_{k}^{a}, w \in V_{a}$ and $q \in E,\|q\|_{\theta} \leq \delta$.

Proof. If the conclusion is false, we can find $a_{m} \rightarrow \infty, k_{m} \geq 2, \theta^{m} \in \Theta_{k_{m}}^{a_{m}}, q_{m} \in E$ with $\left\|q_{m}\right\|_{\theta^{m}} \rightarrow 0$ and $w_{m} \in V_{a_{m}},\left\|w_{m}\right\|_{\theta^{m}}=1$ such that

$$
\left\|\Phi^{\prime \prime}\left(\sum_{i=1}^{k_{m}} \theta_{i}^{m} * q_{0}^{a_{m}}+q_{m}\right) w_{m}\right\|_{\theta^{m}} \rightarrow 0 \quad \text { as } m \rightarrow \infty .
$$

Since $\Phi$ is $\mathbb{Z}$-invariant, we may assume without loss of generality that $\theta_{j_{m}}^{m}=0$ for all $m$, where $j_{m}$ is chosen so that $\left\|w_{m}\right\|_{j_{m}}=\left\|w_{m}\right\|_{\theta^{m}}$. Then $V_{a_{m}}$ is orthogonal to $N(L)^{a_{m}}$. Setting $v_{m}:=\chi_{j_{m}} w_{m}$, where $\chi_{j_{m}}$ is as in Lemma 2.4 gives $\left\|v_{m}\right\| \leq$ $2\left\|w_{m}\right\|_{j_{m}}=2$. Hence $v_{m} \rightarrow w$ in $E$ after passing to a subsequence. Moreover, as $v_{m}=w_{m}$ in $I_{j_{m}}$, it follows that if $z \in N(L)$, then $\left\langle v_{m}, z^{a_{m}}\right\rangle=\left\langle w_{m}, z^{a_{m}}\right\rangle=$ 0 ; therefore $\langle w, z\rangle=0$ for all $z \in N(L)$ and consequently, $w \in R(L)$. Since 
$\left(-a_{m} / 2, a_{m} / 2\right) \subset I_{j_{m}}$ and $a_{m} \rightarrow \infty, w_{m} \rightarrow w$ in $H_{l o c}^{1}\left(\mathbb{R}, \mathbb{R}^{N}\right)$ (i.e., $w_{m} \rightarrow w$ in $H^{1}\left((-a, a), \mathbb{R}^{N}\right)$ for each $\left.a>0\right)$. We also have

$$
\left\langle\Phi^{\prime \prime}\left(\sum_{i=1}^{k_{m}} \theta_{i}^{m} * q_{0}^{a_{m}}+q_{m}\right) w_{m}, v\right\rangle=\left\langle w_{m}, v\right\rangle-\int_{\mathbb{R}} W_{q q}\left(q_{0}^{a_{m}}+q_{m}, t\right) w_{m} \cdot v d t
$$

for each $v \in E, \operatorname{supp} v \subset \widetilde{I}_{j_{m}}$ (the definition of $\widetilde{I}_{j_{m}}$ may be found in Lemma 2.4), and

$$
\lim _{m \rightarrow \infty} \sup _{\substack{\|v\| \leq 1 \\ \operatorname{supp} v \subset \widetilde{I}_{j_{m}}}}\left\langle\Phi^{\prime \prime}\left(\sum_{i=1}^{k_{m}} \theta_{i}^{m} * q_{0}^{a_{m}}+q_{m}\right) w_{m}, v\right\rangle=0 .
$$

According to Lemma 2.3. $\left\|q_{m}\right\|_{\infty} \rightarrow 0$. Hence letting $m \rightarrow \infty$ and using (2.10), (2.11) we obtain $\langle L w, v\rangle=0$ for all $v \in C_{0}^{\infty}\left(\mathbb{R}, \mathbb{R}^{N}\right)$. Therefore $L w=0$, and since $w \in R(L), w=0$. As supp $\chi_{j_{m}} \subset \widetilde{I}_{j_{m}}$, we may insert $v=v_{m}$ in (2.10), so in view of Lemma 2.4 and (2.11),

$$
\liminf _{m \rightarrow \infty} \int_{\mathbb{R}} W_{q q}\left(q_{0}^{a_{m}}+q_{m}, t\right) w_{m} \cdot v_{m} d t \geq \frac{1}{2} .
$$

We complete the proof by showing that this limit must be 0 . Since $q_{0}(t) \rightarrow 0$ as $|t| \rightarrow \infty,\left\|q_{m}\right\|_{\infty} \rightarrow 0$ and $W_{q q}(0, t)=0$, for each $\varepsilon>0$ there exists a bounded interval $I$ such that

$$
\left|\int_{\mathbb{R} \backslash I} W_{q q}\left(q_{0}^{a_{m}}+q_{m}, t\right) w_{m} \cdot v_{m} d t\right| \leq \varepsilon\left\|w_{m}\right\|_{j_{m}}^{2}=\varepsilon
$$

whenever $m$ is large enough. Finally, since $w_{m} \rightarrow 0$ in $H_{l o c}^{1}\left(\mathbb{R}, \mathbb{R}^{N}\right)$, it follows that $w_{m} \rightarrow 0$ in $L^{2}\left(I, \mathbb{R}^{N}\right)$ and

$$
\int_{I} W_{q q}\left(q_{0}^{a_{m}}+q_{m}, t\right) w_{m} \cdot v_{m} d t \rightarrow 0 .
$$

We shall need the following simple observation:

Lemma 2.6. Let $z=z_{1}^{a}+\cdots+z_{k}^{a} \in \bigoplus_{i=1}^{k} \theta_{i} * N(L)^{a}$. Then $\|z\|_{\theta}=\left\|z_{j}^{a}\right\|$, where $\left\|z_{j}^{a}\right\|=\max \left\{\left\|z_{i}^{a}\right\|: 1 \leq i \leq k\right\}$.

Proof. This is an immediate consequence of the fact that the support of each $z_{i}^{a}$ is contained in $I_{i}$.

Recall $P_{a}$ is the orthogonal projection on $V_{a}$; cf. (2.9).

Lemma 2.7. The conclusion of Lemma 2.5 remains valid if $\Phi^{\prime \prime}\left(\sum_{i=1}^{k} \theta_{i} * q_{0}^{a}+q\right) w$ is replaced by $P_{a} \Phi^{\prime \prime}\left(\sum_{i=1}^{k} \theta_{i} * q_{0}^{a}+q\right) w$.

Proof. It suffices to show that if $a_{0}$ is large and $\delta$ small enough, then

$$
\left\|\left(I-P_{a}\right) \Phi^{\prime \prime}\left(\sum_{i=1}^{k} \theta_{i} * q_{0}^{a}+q\right) w\right\|_{\theta} \leq \frac{c}{2}\|w\|_{\theta}
$$

where $c$ is the constant of Lemma 2.5. Suppose $\|w\|_{\theta}=1$, let

$$
\left(I-P_{a}\right) \Phi^{\prime \prime}\left(\sum_{i=1}^{k} \theta_{i} * q_{0}^{a}+q\right) w=: z=z_{1}^{a}+\cdots+z_{k}^{a}, \quad z_{i} \in \theta_{i} * N(L),
$$


and assume without loss of generality that $\left\|z_{j}^{a}\right\|=\max \left\{\left\|z_{i}^{a}\right\|: 1 \leq i \leq k\right\}$ and $\theta_{j}=0$. Then

$$
\begin{aligned}
\left\|z_{j}^{a}\right\|^{2} & =\left\langle z, z_{j}^{a}\right\rangle=\left\langle\left(\Phi^{\prime \prime}\left(\sum_{i=1}^{k} \theta_{i} * q_{0}^{a}+q\right)-\Phi^{\prime \prime}\left(q_{0}\right)\right) w, z_{j}^{a}\right\rangle+\left\langle\Phi^{\prime \prime}\left(q_{0}\right) w, z_{j}^{a}\right\rangle \\
& =-\int_{\mathbb{R}}\left(W_{q q}\left(\sum_{i=1}^{k} \theta_{i} * q_{0}^{a}+q, t\right)-W_{q q}\left(q_{0}, t\right)\right) w \cdot z_{j}^{a} d t+\left\langle\Phi^{\prime \prime}\left(q_{0}\right) w, z_{j}^{a}\right\rangle .
\end{aligned}
$$

Let $\varepsilon>0$ be given. On $I_{j}$ we have $\theta_{i} * q_{0}^{a}(t)=0$ for $i \neq j$ and $\theta_{j} * q_{0}^{a}(t)=q_{0}(t)$ for $|t| \leq a / 8$. Using this and the fact that $q_{0}(t) \rightarrow 0$ as $|t| \rightarrow \infty$, we obtain

$$
\begin{aligned}
& \left|\int_{\mathbb{R}}\left(W_{q q}\left(\sum_{i=1}^{k} \theta_{i} * q_{0}^{a}+q, t\right)-W_{q q}\left(q_{0}, t\right)\right) w \cdot z_{j}^{a} d t\right| \\
& \quad \leq \int_{\mathbb{R}}\left|W_{q q}\left(q_{0}^{a}+q, t\right)-W_{q q}\left(q_{0}, t\right)\right||w|\left|z_{j}^{a}\right| d t \leq \varepsilon\left\|z_{j}^{a}\right\|
\end{aligned}
$$

after choosing $a_{0}$ sufficiently large and $\delta$ sufficiently small. (2.13) will follow if we show that $\left|\left\langle\Phi^{\prime \prime}\left(q_{0}\right) w, z_{j}^{a}\right\rangle\right| \leq \varepsilon\left\|z_{j}^{a}\right\|$. Assuming the contrary, the reverse inequality holds for suitable $a_{m} \rightarrow \infty$ and $w_{m} \in\left[N(L)^{a_{m}}\right]^{\perp},\left\|w_{m}\right\|_{\theta^{m}}=1$. Hence

$$
\left|\left\langle\Phi^{\prime \prime}\left(q_{0}\right) v_{m}, \zeta_{j_{m}}^{a_{m}}\right\rangle\right|=\left|\left\langle\Phi^{\prime \prime}\left(q_{0}\right) w_{m}, \zeta_{j_{m}}^{a_{m}}\right\rangle\right| \geq \varepsilon
$$

where $\zeta_{j_{m}}^{a_{m}}:=z_{j_{m}}^{a_{m}} /\left\|z_{j_{m}}^{a_{m}}\right\|$ and $v_{m}=\chi_{j_{m}} w_{m}$ as in the proof of Lemma 2.5. Passing to a subsequence, $v_{m} \rightarrow w \in R(L), \zeta_{j_{m}}^{a_{m}} \rightarrow \zeta \in N(L)$ and $\left|\left\langle\Phi^{\prime \prime}\left(q_{0}\right) w, \zeta\right\rangle\right| \geq \varepsilon$, which is impossible because $\Phi^{\prime \prime}\left(q_{0}\right) \zeta=L \zeta=0$.

Lemma 2.8. There exists $a_{0} \in \mathbb{N}$ such that if $a \geq a_{0}$, then the mapping $P_{a} \Phi^{\prime \prime}$ : $E_{\theta} \rightarrow \mathcal{L}\left(E_{\theta}\right)$ is uniformly continuous and uniformly bounded on bounded sets. Moreover, the modulus of continuity and the uniform bound are independent of $a$ and $k$.

Proof. We have

$$
\left\langle\left(\Phi^{\prime \prime}(q)-\Phi^{\prime \prime}(\tilde{q})\right) w, v\right\rangle=-\int_{\mathbb{R}}\left(W_{q q}(q, t)-W_{q q}(\tilde{q}, t)\right) w \cdot v d t .
$$

Suppose $\|q\|_{\theta},\|\tilde{q}\|_{\theta} \leq c_{1}$ and let $\varepsilon>0$ be given. Since $W_{q q}$ is uniformly continuous on bounded subsets of $\mathbb{R}^{N} \times \mathbb{R}$ and $\|q\|_{\infty} \leq c\|q\|_{\theta}$ according to Lemma 2.3, there exists $\delta>0$ such that if $\|q-\tilde{q}\|_{\theta} \leq \delta$, then $\left|W_{q q}(q, t)-W_{q q}(\tilde{q}, t)\right| \leq \varepsilon$. Hence it follows from the Sobolev embedding theorem and Lemma 2.4 that if $\left\|\left(\Phi^{\prime \prime}(q)-\Phi^{\prime \prime}(\tilde{q})\right) w\right\|_{\theta}=$ $\left\|\left(\Phi^{\prime \prime}(q)-\Phi^{\prime \prime}(\tilde{q})\right) w\right\|_{j}$ and $v=\chi_{j}\left(\Phi^{\prime \prime}(q)-\Phi^{\prime \prime}(\tilde{q})\right) w /\left\|\left(\Phi^{\prime \prime}(q)-\Phi^{\prime \prime}(\tilde{q})\right) w\right\|_{j}$, then

$$
\left\|\left(\Phi^{\prime \prime}(q)-\Phi^{\prime \prime}(\tilde{q})\right) w\right\|_{j} \leq 2\left\langle\left(\Phi^{\prime \prime}(q)-\Phi^{\prime \prime}(\tilde{q})\right) w, v\right\rangle \leq c_{2} \varepsilon\|w\|_{\theta}
$$

So $\Phi^{\prime \prime}$ is uniformly continuous. To prove that $\Phi^{\prime \prime}$ is uniformly bounded on bounded sets we note that if $\tilde{q}=0$, then (2.14) still holds with $\varepsilon$ replaced by some constant $c_{3}$ which only depends on $c_{1}$.

Now let $\|w\|_{\theta}=1,\left(I-P_{a}\right)\left(\Phi^{\prime \prime}(q)-\Phi^{\prime \prime}(\tilde{q})\right) w=: z=z_{1}^{a}+\cdots+z_{k}^{a}\left(z_{i} \in \theta_{i} * N(L)\right)$ and $\left\|z_{j}^{a}\right\|=\max \left\{\left\|z_{i}^{a}\right\|: 1 \leq i \leq k\right\}$. Then

$$
\left\|z_{j}^{a}\right\|^{2}=\left\langle z, z_{j}^{a}\right\rangle=\left\langle\left(\Phi^{\prime \prime}(q)-\Phi^{\prime \prime}(\tilde{q})\right) w, z_{j}^{a}\right\rangle \leq c_{4} \varepsilon\left\|z_{j}^{a}\right\| .
$$

Hence it follows from Lemma 2.6 that $\left(I-P_{a}\right) \Phi^{\prime \prime}$ is uniformly continuous, and therefore so is $P_{a} \Phi^{\prime \prime}$. Finally, a similar argument shows that $P_{a} \Phi^{\prime \prime}$ is uniformly bounded on bounded sets. 
Let $z=\left(n_{1}, \ldots, n_{k}\right) \in N(L)^{k}, \theta \in \Theta_{k}^{a}, w \in V_{a}$ and consider the mapping

$$
F(\theta, z, w):=P_{a} \Phi^{\prime}\left(\sum_{i=1}^{k} \theta_{i} *\left(q_{0}+n_{i}+v\left(n_{i}\right)\right)^{a}+w\right)
$$

where $v\left(n_{i}\right)$ are as in (2.2). Note that $F(\theta, z, \cdot): V_{a} \rightarrow V_{a}$. Since

$$
F_{w}(\theta, z, 0)=P_{a} \Phi^{\prime \prime}\left(\sum_{i=1}^{k} \theta_{i} *\left(q_{0}+n_{i}+v\left(n_{i}\right)\right)^{a}\right)
$$

and $v\left(n_{i}\right) \rightarrow 0$ as $n_{i} \rightarrow 0$, it follows from Lemma 2.7 that

$$
\left\|F_{w}(\theta, z, 0)^{-1} v\right\|_{\theta} \leq c_{0}\|v\|_{\theta}, \quad v \in V_{a},
$$

where $c_{0}$ is independent of $z, k, \theta$ provided $a$ is large and $\left\|n_{i}\right\|$ are small enough. Further, let

$$
R(z, w):=w-F_{w}(\theta, z, 0)^{-1} F(\theta, z, w)
$$

and note that $R(z, w)=w$ if and only if $F(\theta, z, w)=0$. Our next task will be to solve this equation for $w$.

Lemma 2.9. Given $\delta_{0}>0$, there exist $a_{1} \in \mathbb{N}$ and $\delta \in\left(0, \delta_{0}\right)$ such that if $k \geq 2$, $\left\|n_{i}\right\| \leq \delta(1 \leq i \leq k), a \geq a_{1}$ and $\theta \in \Theta_{k}^{a}$, then $R(z, \cdot)$ is a contraction on the ball $\|w\|_{\theta} \leq \delta$ with respect to the $\theta$-norm.

Proof. Let $c_{0}$ be the constant in (2.16). By Lemma 2.8 and the definition of $F$,

$$
\left\|F_{w}(\theta, z, w)-F_{w}(\theta, z, 0)\right\|_{\mathcal{L}\left(E_{\theta}\right)} \leq \frac{1}{3 c_{0}}
$$

whenever $\left\|n_{i}\right\| \leq \delta,\|w\|_{\theta} \leq \delta$ and $\delta$ is small enough.

Assume without loss of generality that $\|F(\theta, z, 0)\|_{\theta}=\|F(\theta, z, 0)\|_{j}$ and $\theta_{j}=0$. Set

$$
\left(I-P_{a}\right) \Phi^{\prime}\left(\sum_{i=1}^{k} \theta_{i} *\left(q_{0}+n_{i}+v\left(n_{i}\right)\right)^{a}\right)=: y=y_{1}^{a}+\cdots+y_{k}^{a} \quad\left(y_{i} \in \theta_{i} * N(L)\right),
$$

let $\chi_{j}$ be as in Lemma 2.4 and let $w:=F(\theta, z, 0) /\|F(\theta, z, 0)\|_{j}$. Then $w \in V_{a}$, hence $w \in\left[N(L)^{a}\right]^{\perp}$, and by Lemma 2.4 and (2.15),

$$
\begin{gathered}
\frac{1}{2}\|F(\theta, z, 0)\|_{\theta} \leq\left\langle F(\theta, z, 0), \chi_{j} w\right\rangle=\left\langle P_{a} \Phi^{\prime}\left(\sum_{i=1}^{k} \theta_{i} *\left(q_{0}+n_{i}+v\left(n_{i}\right)\right)^{a}\right), \chi_{j} w\right\rangle \\
=\left\langle\Phi^{\prime}\left(\sum_{i=1}^{k} \theta_{i} *\left(q_{0}+n_{i}+v\left(n_{i}\right)\right)^{a}\right), \chi_{j} w\right\rangle-\left\langle y_{1}^{a}+\cdots+y_{k}^{a}, \chi_{j} w\right\rangle \\
=\left\langle\Phi^{\prime}\left(\left(q_{0}+n_{j}+v\left(n_{j}\right)\right)^{a}\right), w\right\rangle
\end{gathered}
$$

(we have used that $y_{j} \in N(L)^{a}, \chi_{j}=1$ in $I_{j}$ and $\chi_{j}=0$ off $\widetilde{I}_{j}$ ). We claim

$$
\|F(\theta, z, 0)\|_{\theta} \rightarrow 0 \text { uniformly in } k, z, \theta \text { as } a \rightarrow \infty \text {. }
$$

Arguing by contradiction, we find $\varepsilon>0, a_{m} \rightarrow \infty, k_{m} \geq 2, z^{m} \in N(L)^{k_{m}}$, $\theta^{m} \in \Theta_{k_{m}}^{a_{m}}$ and $w_{m} \in\left[N(L)^{a_{m}}\right]^{\perp},\left\|w_{m}\right\|_{\theta^{m}}=1$, such that

$$
\varepsilon \leq \frac{1}{2}\left\|F\left(\theta^{m}, z^{m}, 0\right)\right\|_{\theta^{m}} \leq\left\langle\Phi^{\prime}\left(\left(q_{0}+n_{j_{m}}+v\left(n_{j_{m}}\right)\right)^{a_{m}}\right), w_{m}\right\rangle .
$$


Since $w_{m}=\chi_{j_{m}} w_{m}$ in $I_{j_{m}}, \chi_{j_{m}} w_{m} \rightarrow w \in R(L)$ and $n_{j_{m}} \rightarrow n$ after passing to a subsequence, it follows using (2.2) and (2.5) that $\varepsilon \leq\left\langle\Phi^{\prime}\left(q_{0}+n+v(n)\right), w\right\rangle=0$, a contradiction. Hence (2.18) holds and in particular,

$$
\|F(\theta, z, 0)\|_{\theta} \leq \frac{\delta}{3 c_{0}}
$$

if $a$ is large enough $\left(a \geq a_{1}, k \geq 2, \theta \in \Theta_{k}^{a}\right)$. Fix $k \geq 2, \theta \in \Theta_{k}^{a}$ and let $\|w\|_{\theta} \leq \delta$. Then

$$
\begin{aligned}
R(z, w)= & -F_{w}(\theta, z, 0)^{-1} F(\theta, z, 0) \\
& -F_{w}(\theta, z, 0)^{-1}\left(F(\theta, z, w)-F(\theta, z, 0)-F_{w}(\theta, z, 0) w\right),
\end{aligned}
$$

and using (2.16), (2.17), and (2.19),

$$
\begin{aligned}
\|R(z, w)\|_{\theta} \leq & \left\|F_{w}(\theta, z, 0)^{-1}\right\|_{\mathcal{L}\left(V_{a, \theta}\right)}\|F(\theta, z, 0)\|_{\theta} \\
& +\left\|F_{w}(\theta, z, 0)^{-1}\right\|_{\mathcal{L}\left(V_{a, \theta}\right)}\left\|F(\theta, z, w)-F(\theta, z, 0)-F_{w}(\theta, z, 0) w\right\|_{\theta} \\
\leq & \frac{\delta}{3}+c_{0} \int_{0}^{1}\left\|F_{w}(\theta, z, s w)-F_{w}(\theta, z, 0)\right\|_{\mathcal{L}\left(V_{a, \theta}\right)}\|w\|_{\theta} d s \leq \frac{\delta}{3}+c_{0} \frac{\delta}{3 c_{0}}
\end{aligned}
$$

$\left(\mathcal{L}\left(V_{a, \theta}\right)\right.$ is the space of bounded linear operators on $V_{a}$ with the $\theta$-norm). Hence $\|R(z, w)\|_{\theta} \leq \delta$. Furthermore, if $\|w\|_{\theta},\|\tilde{w}\|_{\theta} \leq \delta$, then

$$
\begin{aligned}
\| R(z, & w)-R(z, \tilde{w}) \|_{\theta} \\
& \leq\left\|F_{w}(\theta, z, 0)^{-1}\right\|_{\mathcal{L}\left(V_{a, \theta}\right)}\left\|F(\theta, z, w)-F(\theta, z, \tilde{w})-F_{w}(\theta, z, 0)(w-\tilde{w})\right\|_{\theta} \\
& \leq c_{0} \int_{0}^{1}\left\|F_{w}(\theta, z, s w+(1-s) \tilde{w})-F_{w}(\theta, z, 0)\right\|_{\mathcal{L}\left(V_{a, \theta}\right)}\|w-\tilde{w}\|_{\theta} d s \\
& \leq \frac{1}{3}\|w-\tilde{w}\|_{\theta},
\end{aligned}
$$

so $R(z, \cdot)$ is a contraction on $\|w\|_{\theta} \leq \delta$.

Proposition 2.10. Given $\delta_{0}>0$, there exist $a_{1} \in \mathbb{N}$ and $\delta \in\left(0, \delta_{0}\right)$ such that if $k \geq 2,\left\|n_{i}\right\| \leq \delta(1 \leq i \leq k), a \geq a_{1}$ and $\theta \in \Theta_{k}^{a}$, then there is a unique $w=w(\theta, z) \in V_{a}$ such that $F(\theta, z, w(\theta, z))=0$ and $\|w(\theta, z)\|_{\theta} \leq \delta$. Moreover, $w(\theta, z)$ is of class $C^{1}$ and $\|w(\theta, z)\|_{\theta} \rightarrow 0$ uniformly in $k, z, \theta$ as $a \rightarrow \infty$.

Proof. Existence and uniquess of $w$ follows from the contraction mapping principle and the preceding lemma. Moreover, $\|w(\theta, z)\|_{\theta} \leq \delta$ because $\|R(z, w)\|_{\theta} \leq \delta$. Since $F_{w}(\theta, z, w(\theta, z))$ is invertible on $V_{a}$ according to (2.16) and (2.17), $w(\theta, z)$ is of class $C^{1}$ by the implicit function theorem.

Set $w=w(\theta, z)$ and $\|R(z, 0)\|_{\theta}=\varepsilon$; then by (2.20), $\|R(z, w)\|_{\theta} \leq\|R(z, 0)\|_{\theta}+$ $\frac{1}{3}\|w\|_{\theta} \leq \varepsilon+\frac{\delta}{3}$. So $\|w\|_{\theta} \leq \varepsilon+\frac{\delta}{3}$ and $\|R(z, w)\|_{\theta} \leq\left(1+\frac{1}{3}\right) \varepsilon+\frac{\delta}{9}$. Iterating this procedure we see that $\|w\|_{\theta} \leq \frac{3}{2}\|R(z, 0)\|_{\theta}$, and it follows from (2.16) and (2.18) that

$$
\|R(z, 0)\|_{\theta} \leq\left\|F_{w}(\theta, z, 0)^{-1}\right\|_{\mathcal{L}\left(V_{a, \theta}\right)}\|F(\theta, z, 0)\|_{\theta} \rightarrow 0 \text { as } a \rightarrow \infty .
$$

Hence $\|w\|_{\theta} \rightarrow 0$ as well. 
Suppose now $a_{1}$ and $\delta$ are chosen as in Proposition 2.10 and let

$$
\widetilde{\varphi}(z):=\Phi\left(\sum_{i=1}^{k} \theta_{i} *\left(q_{0}+n_{i}+v\left(n_{i}\right)\right)^{a}+w(\theta, z)\right) .
$$

Then $\widetilde{\varphi}$ is well defined on the set $D_{\delta}:=\left\{z=\left(n_{1}, \ldots, n_{k}\right) \in N(L)^{k}:\left\|n_{i}\right\| \leq \delta\right\}$. Denote $\zeta=\sum_{i=1}^{k} \theta_{i} *\left(q_{0}+n_{i}+v\left(n_{i}\right)\right)^{a}+w(\theta, z)$ and let $y=\left(m_{1}, \ldots, m_{k}\right) \in N(L)^{k}$. Since $F(\theta, z, w(\theta, z))=P_{a} \Phi^{\prime}(\zeta)=0$ and $w^{\prime}(\theta, z)$ maps into $V_{a}$, we see that

$$
\begin{aligned}
\widetilde{\varphi}^{\prime}(z) \cdot y & =\left\langle\Phi^{\prime}(\zeta), \sum_{i=1}^{k} \theta_{i} *\left(m_{i}+v^{\prime}\left(n_{i}\right) m_{i}\right)^{a}+w^{\prime}(\theta, z) y\right\rangle \\
& =\left\langle\left(I-P_{a}\right) \Phi^{\prime}(\zeta), \sum_{i=1}^{k} \theta_{i} *\left(m_{i}+v^{\prime}\left(n_{i}\right) m_{i}\right)^{a}\right\rangle .
\end{aligned}
$$

Lemma 2.11. Given $\delta_{0}>0$, there exist $a_{1} \in \mathbb{N}$ and $\delta \in\left(0, \delta_{0}\right)$ such that whenever $k \geq 2, a \geq a_{1}, \theta \in \Theta_{k}^{a}$ and $z \in D_{\delta}$, then $\widetilde{\varphi}^{\prime}(z)=0$ if and only if $\zeta=\sum_{i=1}^{k} \theta_{i} *$ $\left(q_{0}+n_{i}+v\left(n_{i}\right)\right)^{a}+w(\theta, z)$ is a solution of (1.1).

Proof. It is clear that if $\zeta$ is a solution of (1.1), then $\widetilde{\varphi}^{\prime}(z)=0$. Suppose $\widetilde{\varphi}^{\prime}(z)=0$ and let $\left(I-P_{a}\right) \Phi^{\prime}(\zeta)=\xi_{1}^{a}+\ldots+\xi_{k}^{a}$, where $\xi_{i} \in \theta_{i} * N(L), i=1, \ldots, k$. We shall show that $\xi_{1}=\ldots=\xi_{k}=0$. Assume without loss of generality that $\left\|\xi_{1}^{a}\right\|=$ $\max \left\{\left\|\xi_{i}^{a}\right\|: 1 \leq i \leq k\right\}, \theta_{1}=0$ and let $m_{1}=\xi_{1}, m_{2}=\ldots=m_{k}=0$. Then

$$
0=\left\langle\xi_{1}^{a}+\ldots+\xi_{k}^{a},\left(\xi_{1}+v^{\prime}\left(n_{1}\right) \xi_{1}\right)^{a}\right\rangle=\left\|\xi_{1}^{a}\right\|^{2}+\left\langle\xi_{1}^{a},\left(v^{\prime}\left(n_{1}\right) \xi_{1}\right)^{a}\right\rangle,
$$

and since $v^{\prime}\left(n_{1}\right) \xi_{1} \in R(L),\left|\left\langle\xi_{1}^{a},\left(v^{\prime}\left(n_{1}\right) \xi_{1}\right)^{a}\right\rangle\right| \leq \frac{1}{2}\left\|\xi_{1}^{a}\right\|^{2}$ provided $a$ is large enough. Hence $\xi_{1}^{a}=0$.

To complete the proof of Theorem 1.1 we must show $\widetilde{\varphi}$ has a critical point in $D_{\delta}$. Recall that $\varphi(n):=\Phi\left(q_{0}+n+v(n)\right)$ and choose $\delta_{0}$ so that 0 is the only critical point of $\varphi$ in the set $\|n\| \leq \delta_{0}$. Let $a_{1}$ and $\delta$ be as in the conclusion of Lemma 2.11, let $Y$ be a pseudogradient vector field for $\varphi$ and $\left(W, W^{-}\right)$a corresponding GromollMeyer pair contained in the ball $\|n\|<\delta$ (see [10] for the definition of a GromollMeyer pair). Here it will be convenient to use a definition of the pseudogradient field which somewhat differs from the usual one (cf. 21]): we require that $Y$ be defined and locally Lipschitz continuous except at the critical set of $\varphi,\|Y\| \leq 1$ and $\left\langle\varphi^{\prime}(u), Y(u)\right\rangle$ is positive and bounded away from 0 on all subsets which are bounded away from the critical set of $\varphi$.

We shall use the customary notation

$$
(A, C) \times(B, D):=(A \times B, A \times D \cup C \times B),
$$

and we set

$$
\left(\widetilde{W}, \widetilde{W}^{-}\right):=\left(W, W^{-}\right)^{k} \equiv \underbrace{\left(W, W^{-}\right) \times \ldots \times\left(W, W^{-}\right)}_{k \text { times }} .
$$

Note that $\widetilde{W} \subset D_{\delta}$ and consider the flow $\eta$ given by

$$
\frac{d \eta}{d s}=-\chi(\eta) Y(\eta), \quad \eta(0, n)=n \quad(n \in N(L),\|n\| \leq \delta)
$$

where $\chi \in C^{\infty}\left(D_{\delta},[0,1]\right)$ is such that $\chi=0$ close to $n=0$ and $\chi=1$ close to the boundary of $W$. 
Proposition 2.12. There exists $a_{2} \geq a_{1}$ such that if $a \geq a_{2}$, then $\left(\widetilde{W}, \widetilde{W}^{-}\right)$is a Gromoll-Meyer pair for $\widetilde{\varphi}$ and the (possibly empty) set of critical points contained in the interior of $\widetilde{W}$.

Proof. Let $z=\left(n_{1}, \ldots, n_{k}\right)$ and $\tilde{Y}(z):=\left(\chi\left(n_{1}\right) Y\left(n_{1}\right), \ldots, \chi\left(n_{k}\right) Y\left(n_{k}\right)\right)$. The flow of $-\widetilde{Y}$ is $\widetilde{\eta}(s, z)=\left(\eta\left(s, n_{1}\right), \ldots, \eta\left(s, n_{k}\right)\right)$. It is therefore clear that $\widetilde{\eta}$ can leave $\widetilde{W}$ only via $\widetilde{W}^{-}$and that $\widetilde{\eta}(\cdot, z)$ has the required transversality property on $\widetilde{W}^{-}$. We shall show that $\tilde{Y} /\|\widetilde{Y}\|$ is a pseudogradient field in a neighborhood of the boundary of $\widetilde{W}$. Using a partition of unity, $\widetilde{Y}$ can be modified in the interior of $\widetilde{W}$ in such a way that it becomes a pseudogradient field on the whole of $\widetilde{W}$, except at critical points. This will complete the proof.

Let $y=\left(m_{1}, 0, \ldots, 0\right), m_{1} \in N(L)$ and assume without loss of generality that $\theta_{1}=0$. Then by (2.21) and since $P_{a} \Phi^{\prime}(\zeta)=0$,

$$
\begin{aligned}
& \widetilde{\varphi}^{\prime}(z) \cdot y=\left\langle\left(I-P_{a}\right) \Phi^{\prime}(\zeta),\left(m_{1}+v^{\prime}\left(n_{1}\right) m_{1}\right)^{a}\right\rangle=\left\langle\Phi^{\prime}(\zeta),\left(m_{1}+v^{\prime}\left(n_{1}\right) m_{1}\right)^{a}\right\rangle \\
& =\left\langle\Phi^{\prime}\left(\left(q_{0}+n_{1}+v\left(n_{1}\right)\right)^{a}+w(\theta, z)\right),\left(m_{1}+v^{\prime}\left(n_{1}\right) m_{1}\right)^{a}\right\rangle \\
& =\left\langle\Phi^{\prime}\left(\left(q_{0}+n_{1}+v\left(n_{1}\right)\right)^{a}\right),\left(m_{1}+v^{\prime}\left(n_{1}\right) m_{1}\right)^{a}\right\rangle \\
& \quad+\left\langle\Phi^{\prime}\left(\left(q_{0}+n_{1}+v\left(n_{1}\right)\right)^{a}+w(\theta, z)\right)\right. \\
& \left.\quad-\Phi^{\prime}\left(\left(q_{0}+n_{1}+v\left(n_{1}\right)\right)^{a}\right),\left(m_{1}+v^{\prime}\left(n_{1}\right) m_{1}\right)^{a}\right\rangle .
\end{aligned}
$$

Since $\left(q_{0}+n_{1}+v\left(n_{1}\right)\right)^{a} \rightarrow q_{0}+n_{1}+v\left(n_{1}\right)$ uniformly in $n_{1}$ as $a \rightarrow \infty$ and $Y$ is a pseudogradient vector field for $\varphi$ on $W$, we see from (2.4) that if we choose $m_{1}=Y\left(n_{1}\right)$, then the first term on the right-hand side above is larger than or equal to $2 \varepsilon$ for some $\varepsilon>0$ whenever $a$ is large enough $\left(a \geq a_{2}\right)$ and $n_{1}$ is close to $W^{-}$. Moreover, $\varepsilon$ is independent of the particular choice of $a, k$ and $\theta$. Since $\|w(\theta, z)\|_{\theta} \rightarrow$ 0 as $a \rightarrow \infty$, it is easy to see that the absolute value of the second term is less than or equal to $\varepsilon$, possibly after choosing a larger $a_{2}$. Hence $\widetilde{\varphi}^{\prime}(z) \cdot\left(Y\left(n_{1}\right), 0, \ldots, 0\right) \geq \varepsilon$ for $n_{1}$ close to the boundary of $W$. Of course, the same argument applies to any $y=\left(0, \ldots, 0, Y\left(n_{j}\right), 0, \ldots, 0\right)$, where $a_{2}$ and $\varepsilon$ can easily be made independent of $j$. Since $\|\widetilde{Y}\|$ is bounded, $\widetilde{\varphi}^{\prime}(z) \cdot \tilde{Y}(z) /\|\widetilde{Y}(z)\|$ is bounded away from 0 in a neighborhood of $\widetilde{W}^{-}$(this bound is allowed to depend on $k$ ). Hence $\left(\widetilde{W}, \widetilde{W}^{-}\right)$is a Gromoll-Meyer pair.

Proof of Theorem 1.1. By a result of Dancer [16] we may assume $\left(W, W^{-}\right)$is a pair of ANR's. Hence $\left(\widetilde{W}, \widetilde{W}^{-}\right)=\left(W, W^{-}\right)^{k}$ satisfies the excision requirement of Künneth's formula and therefore (see [19, Corollary VI.12.12])

$$
H_{*}\left(\widetilde{W}, \widetilde{W}^{-}\right)=H_{*}\left(\left(W, W^{-}\right)^{k}\right)=H_{*}\left(W, W^{-}\right) \otimes \cdots \otimes H_{*}\left(W, W^{-}\right) .
$$

Since $H_{r}\left(W, W^{-}\right) \neq 0$ for some $r$ according to Lemma 2.1, $H_{k r}\left(\widetilde{W}, \widetilde{W}^{-}\right) \neq 0$. It follows that $\widetilde{\varphi}$ has a critical point in $\widetilde{W}$ (for otherwise a standard argument shows $\widetilde{W}^{-}$is a deformation retract of $\widetilde{W}$ and hence $\left.H_{*}\left(\widetilde{W}, \widetilde{W}^{-}\right)=0\right)$.

We have shown that there exists a solution $\bar{q}$ of (1.1) which is of the form

$$
\bar{q}=\sum_{i=1}^{k} \theta_{i} * q_{0}+v,
$$


where

$$
v=\sum_{i=1}^{k} \theta_{i} *\left(q_{0}^{a}-q_{0}\right)+\sum_{i=1}^{k} \theta_{i} *\left(n_{i}+v\left(n_{i}\right)\right)^{a}+w(\theta, z) .
$$

We complete the proof by showing that if $a_{0}$ is large enough, then $\|v\|_{\infty} \leq c \delta_{0}$, where $c$ is a constant independent of the particular choice of $a, k$ and $\theta$. In (2.23) we have $\|w(\theta, z)\|_{\theta} \leq \delta_{0}$ and $\left\|\sum_{i=1}^{k} \theta_{i} *\left(n_{i}+v\left(n_{i}\right)\right)^{a}\right\|_{\theta} \leq 2 \delta_{0}$ provided $\delta_{0}$ is small enough (cf. Proposition 2.10 and recall $v(0)=0, v^{\prime}(0)=0$ ). Since 0 is a hyperbolic point for (1.1), $\left|q_{0}(t)\right|,\left|\dot{q}_{0}(t)\right| \leq c_{1} e^{-\alpha|t|}$ for some $c_{1}, \alpha>0$ (see Remark 2.14 below). According to (2.6) and (2.7), the distance from $\theta_{j}$ to $I_{i}$ is larger than or equal to $a(|i-j|-1 / 2)$ whenever $i \neq j$; hence

$$
\left\|\theta_{i} *\left(q_{0}^{a}-q_{0}\right)\right\|_{j}=\left\|\theta_{i} * q_{0}\right\|_{j} \leq c_{2} e^{-\alpha a(|i-j|-1 / 2)}, \quad i \neq j,
$$

and

$$
\left\|\theta_{j} *\left(q_{0}^{a}-q_{0}\right)\right\|_{j} \leq c_{2} e^{-\alpha a}
$$

for a suitable $c_{2}>0$. Summing over all $i$ we see that $\left\|\sum_{i=1}^{k} \theta_{i} *\left(q_{0}^{a}-q_{0}\right)\right\|_{\theta} \leq \delta_{0}$ whenever $a$ is large enough. Hence $\|v\|_{\infty} \leq c_{3}\|v\|_{\theta} \leq c \delta_{0}$.

Remark 2.13. If $N(L)=\{0\}$ (i.e., $q_{0}$ is a nondegenerate critical point), the proof of Theorem 1.1 becomes much simpler. Since $V_{a}=E$ and $P_{a}$ is the identity mapping, we now have

$$
F=F(\theta, w)=\Phi^{\prime}\left(\sum_{i=1}^{k} \theta_{i} * q_{0}^{a}+w\right)
$$

(cf. (2.15)), and with the aid of Lemmas 2.5 and 2.8 we see that $R=R(w)(w \in E$, $\left.\|w\|_{\theta} \leq \delta\right)$ is a contraction. Hence there is a unique $w=w(\theta)$ such that

$$
F(\theta, w(\theta))=\Phi^{\prime}\left(\sum_{i=1}^{k} \theta_{i} * q_{0}^{a}+w(\theta)\right)=0
$$

Moreover, the solution $\bar{q}=\sum_{i=1}^{k} \theta_{i} * q_{0}+v$ is nondegenerate. The case $N(L)=\{0\}$ was considered earlier in [4]. Note that the nondegeneracy of $q_{0}$ implies $C_{r}\left(\Phi, q_{0}\right)=$ $\delta_{M^{-}(L)}^{r} \mathcal{F}$ (this fact has not been used here).

Remark 2.14. Although it is rather well known that $\left|q_{0}(t)\right|,\left|\dot{q}_{0}(t)\right| \leq c_{1} e^{-\alpha|t|}$ for some $c_{1}, \alpha>0$, we sketch the proof for the reader's convenience. We note that $q_{0}$ is a homoclinic solution for the linear system

$$
-\ddot{q}+(B(t)-C(t)) q=0,
$$

where $c_{i j}(t):=W_{q_{i}}\left(q_{0}(t), t\right) q_{0, j}(t) /\left|q_{0}(t)\right|^{2}$ are the entries of the matrix $C$ and $q_{0}=\left(q_{0,1}, \ldots, q_{0, N}\right)$. Setting $p=\dot{q}$ we get the first order system

$$
\dot{p}=(B(t)-C(t)) q, \quad \dot{q}=p,
$$

in $\mathbb{R}^{2 N}$. Since $B$ is positive definite, (2.24) with $C=0$ has an exponential dichotomy according to [11, Proposition 6.1] (one can e.g. choose $\alpha=\sqrt{\beta} / 2$, where $\beta$ is such that $B(t) q \cdot q \geq \beta|q|^{2}$ for all $\left.q \in \mathbb{R}^{N}, t \in \mathbb{R}\right)$. As $c_{i j}(t) \rightarrow 0$ as $|t| \rightarrow \infty$, it follows by roughness of dichotomies [11, Proposition 4.1] and by [11, p. 13] that also the full system (2.24) has an exponential dichotomy. Hence $q_{0}$ and $\dot{q}_{0}$ have the decay as claimed. 


\section{Corollaries to Theorem 1.1}

By applying the same arguments as in the preceding section we immediately obtain the following more general result:

Corollary 3.1. Suppose the hypotheses $\left(H_{1}\right)-\left(H_{3}\right)$ are satisfied and $q_{j}, 1 \leq j \leq m$, are isolated homoclinic solutions of (1.1) such that $C_{*}\left(\Phi, q_{j}\right) \neq 0$ for all $j$. For each $\delta>0$ there exists $a \in \mathbb{N}$ with the property that if $k \geq 2, \theta_{1}, \theta_{2}, \ldots, \theta_{k} \in \mathbb{Z}$ and $\theta_{i+1}-\theta_{i} \geq a$ for all $i=1,2, \ldots, k-1$, then there $i s v \in E$ such that $\|v\|_{\infty} \leq \delta$ and $\bar{q}=\theta_{1} * q_{j_{1}}+\cdots+\theta_{k} * q_{j_{k}}+v$, where $j_{1}, \ldots, j_{k} \in\{1, \ldots, m\}$, is a homoclinic solution of (1.1).

As a simple example we may consider (1.1) with $W$ even in $q$. If $q_{0}$ is an isolated homoclinic such that $C_{*}\left(\Phi, q_{0}\right) \neq 0$, then we may take $q_{1}=q_{0}$ and $q_{2}=-q_{0}$ in the above corollary.

In Theorem 1.1 we have shown that for each choice of $\theta_{1}, \ldots, \theta_{m}$ with $\theta_{i+1}-\theta_{i}$ large enough there exists an $m$-bump solution. Since $H_{*}\left(\widetilde{W}, \widetilde{W}^{-}\right) \neq 0$ as has been seen in the proof, it is not very restrictive to assume that there exists an isolated $m$-bump solution $q_{m}$ with $C_{*}\left(\Phi, q_{m}\right) \neq 0$. By Corollary 3.1 it is then possible to obtain a $k$-bump solution $\bar{q}$ such that each $q_{j_{i}}, 1 \leq i \leq k$, is an $m_{i}$-bump solution obtained from $q_{0}$. In other words, we can glue $m_{i}$-bump solutions into a new multibump solution. However, we do not know whether it necessarily differs from all $m_{1}+\cdots+m_{k}$-bump solutions obtained with the aid of Theorem 1.1 .

Next we discuss the existence of periodic $k$-bump solutions of arbitrarily large minimal period $T$. Let $\theta_{0}, \theta_{k+1}$ be finite and let $\Theta_{k}^{a}$ be as in (2.6) except that now we also include $\theta_{0}$ and $\theta_{k+1}$ in $\theta$. Then all intervals $I_{i}$ in (2.7) are finite. Let $J:=\left(\frac{1}{2}\left(\theta_{0}+\theta_{1}\right), \frac{1}{2}\left(\theta_{k}+\theta_{k+1}\right)\right), T:=|J|($ the length of the interval $J)$ and

$$
E_{T}:=\left\{q \in H^{1}\left(J, \mathbb{R}^{N}\right): q\left(\frac{1}{2}\left(\theta_{0}+\theta_{1}\right)\right)=q\left(\frac{1}{2}\left(\theta_{k}+\theta_{k+1}\right)\right)\right\} .
$$

In $E_{T}$ we use the inner product and the norm (1.2) except that we integrate over $J$ instead of $\mathbb{R}$. Obviously, $\Phi$ can be defined on $E_{T}$ by the formula (1.3), where again $\mathbb{R}$ is replaced by $J$, and we may assume the functions $q \in E_{T}$ have been extended periodically. Then $\Phi \in C^{2}\left(E_{T}, \mathbb{R}\right)$ and $\Phi^{\prime}(q)=0$ if and only if $q$ is a $T$-periodic solution of (1.1). We may also introduce the $\theta$-norm (2.8) on $E_{T}$.

Corollary 3.2. Suppose the hypotheses $\left(H_{1}\right)-\left(H_{3}\right)$ are satisfied and $q_{0}$ is an isolated homoclinic solution of (1.1) such that $C_{*}\left(\Phi, q_{0}\right) \neq 0$. For each $\delta>0$ there exists $a \in \mathbb{N}$ with the property that if $k \geq 1, \theta_{0}, \theta_{1}, \ldots, \theta_{k+1} \in \mathbb{Z}$ and $\theta_{i+1}-\theta_{i} \geq$ a for all $i=0,1, \ldots, k$, then there is $v \in H^{1}\left(J, \mathbb{R}^{N}\right),\|v\|_{\infty} \leq \delta$, and a T-periodic solution $\bar{q}$ of (1.1) such that $\bar{q}=\theta_{1} * q_{0}+\cdots+\theta_{k} * q_{0}+v$ for $t \in J$.

Note that if $k=1$, then $J=I_{1}$ and $T=\left|I_{1}\right|$ is the minimal period of $\bar{q}$ for reasonably small $\delta$. If $k \geq 2$, then in general $T$ may not be the minimal period; however, this must be the case if some $I_{i_{0}}$ is larger than the other intervals and $\delta$ is sufficiently small.

Since the proof of Corollary 3.2 parallels that of Theorem 1.1, we only point out the main differences. A general difference is that integration over $\mathbb{R}$ should be replaced by integration over $J$ throughout. Lemmas 2.3 and 2.4 remain valid except that in the definition of $\widetilde{I}_{j}$ the intervals $I_{1}$ and $I_{k}$ are considered to be adjacent. Since $\bigoplus_{i=1}^{k} \theta_{i} * N(L)^{a} \subset E_{T}$, it makes sense to define $V_{T, a}$ and $P_{T, a}$ as in (2.9), with the orthogonal complement taken in $E_{T}$. In Lemma 2.5 $E$ should be replaced by $E_{T}$ 
and $V_{a}$ by $V_{T, a}$. In the argument, by contradiction $q_{m} \in E_{T_{m}}$ and $w_{m} \in V_{T_{m}, a_{m}}$. By the $T_{m}$-periodicity we may assume that $I_{j_{m}}$ is neither the first nor the last interval in $J_{m}$; therefore $v_{m}=\chi_{j_{m}} w_{m}$ can be considered both as an element of $E_{T_{m}}$ and of $E$. It follows that $w \in R(L)$ and, moreover, the inner product in (2.10) and (2.11) is the same in $E$ and in $E_{T_{m}}$ if $\operatorname{supp} v \subset \widetilde{I}_{j_{m}}$. Now Lemma 2.5 follows, and so do Lemmas 2.7 and 2.8 after taking the above modifications into account. The mapping $F$ is defined as in (2.15), except that $w \in V_{T, a}$ and $P_{a}$ is replaced by $P_{T, a}$. So $F: V_{T, a} \rightarrow V_{T, a}$. The remaining steps in the proof of Theorem 1.1 go through after making easy changes in line with the modifications described above. In particular, the definition of $\widetilde{\varphi}$ is the same except that $w(\theta, z) \in V_{T, a}$. Note that although $w(\theta, z) \in V_{T, a} \subset E_{T}$ and $\bar{q} \in E_{T}$, $v$, which is given by (2.23), need not be in $E_{T}$ (because in general $q_{0}^{a}-q_{0} \notin E_{T}$ ).

We conclude this section by showing that there exist solutions having infinitely many bumps. The argument we present here is well known [30].

Corollary 3.3. Suppose the hypotheses $\left(H_{1}\right)-\left(H_{3}\right)$ are satisfied and $q_{0}$ is an isolated homoclinic solution of (1.1) such that $C_{*}\left(\Phi, q_{0}\right) \neq 0$. For each $\delta>0$ there exists $a \in$ $\mathbb{N}$ with the property that if $\left(\theta_{i}\right)_{i \in \mathbb{Z}}$ is a sequence such that $\theta_{i} \in \mathbb{Z}$ and $\theta_{i+1}-\theta_{i} \geq$ a for all $i \in \mathbb{Z}$, then there is $v \in H_{\text {loc }}^{1}\left(\mathbb{R}, \mathbb{R}^{N}\right)$ such that $\|v\|_{\infty} \leq \delta$ and $\bar{q}=\sum_{i \in \mathbb{Z}} \theta_{i} * q_{0}+v$ is a solution of (1.1). A similar conclusion holds if $\left(\theta_{i}\right)_{i \in \mathbb{Z}}$ is replaced by $\left(\theta_{i}\right)_{i \in \mathbb{N}}$ or $\left(\theta_{i}\right)_{i \in-\mathbb{N}}$.

Note that $\bar{q}$ above is not a homoclinic solution and it does not need to be periodic.

Proof. We consider the case $i \in \mathbb{Z}$, the other ones being similar. Let $\delta$ and a corresponding $a$ be as in Theorem 1.1. For each $m \geq 1$ there exists a homoclinic solution

$$
q^{m}(t)=\sum_{i=-m}^{m} \theta_{i} * q_{0}(t)+v^{m}(t)
$$

such that $\left\|v^{m}\right\|_{\infty} \leq \delta$. Since $q_{0}$ decays exponentially, $\left\|q^{m}\right\|_{\infty}$ is bounded uniformly in $m$; therefore it follows from (1.1) that so is $\left\|\ddot{q}^{m}\right\|_{\infty}$, and a posteriori, $\left\|\ddot{v}^{m}\right\|_{\infty}$. By the Arzelà-Ascoli theorem, for each $M \in \mathbb{N}$ there exists $v$ such that $v^{m} \rightarrow v$ in $L^{\infty}\left((-M, M), \mathbb{R}^{N}\right)$ after passing to a subsequence. Using the diagonal procedure we can define $v$ on $\mathbb{R}$ in such a way that, up to a subsequence, $v^{m} \rightarrow v$ in $L_{\text {loc }}^{\infty}\left(\mathbb{R}, \mathbb{R}^{N}\right)$ and $\|v\|_{\infty} \leq \delta$. Then the corresponding function $\bar{q}$ is a weak solution of (1.1). By standard regularity theory $\bar{q}$ is a classical solution and in particular, $\bar{q} \in H_{l o c}^{1}\left(\mathbb{R}, \mathbb{R}^{N}\right)$.

It is clear from the above proofs that the conclusions of Corollaries 3.2 and 3.3 remain valid in the framework of Corollary 3.1

\section{Relation to the Bernoulli shift}

First we recall the definition of the Bernoulli shift. Let

$$
\Sigma_{2}:=\{0,1\}^{\mathbb{Z}}=\left\{s=\left\{s_{j}\right\}_{j \in \mathbb{Z}}: s_{j} \in\{0,1\}\right\}
$$

be the set of doubly infinite sequences of 0 's and 1's, endowed with the metric

$$
d(s, \tilde{s}):=\sum_{j \in \mathbb{Z}} 2^{-|j|}\left|s_{j}-\tilde{s}_{j}\right| .
$$


$\left(\Sigma_{2}, d\right)$ is a compact and totally disconnected space, homeomorphic to the Cantor set. The mapping $\sigma: \Sigma_{2} \rightarrow \Sigma_{2}$ given by

$$
(\sigma(s))_{j}=s_{j+1}
$$

is called the Bernoulli shift on two symbols. It is continuous, it has a countable infinity of periodic orbits, an uncountable infinity of nonperiodic orbits, a dense orbit and exhibits sensitive dependence on initial conditions; see e.g. 35, Chapter 2 ] for more details. Moreover, the topological entropy $h(\sigma)$ equals $\log 2$ [33, Example 5.8.4] and is thus positive.

Let $M$ be a metric space and $f: M \rightarrow M, g: M \rightarrow \Sigma_{2}$ continuous mappings. We shall say $(M, f)$ is semiconjugate to $\left(\Sigma_{2}, \sigma\right)$ if $g$ is surjective and the diagram

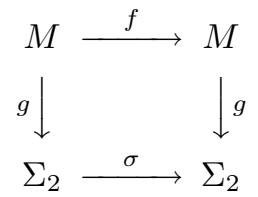

is commutative. If $g$ is a homeomorphism, then $f$ is conjugate to $\sigma$. It can be shown that $h(f) \geq h(\sigma)$ whenever $(M, f)$ is semiconjugate to $\left(\Sigma_{2}, \sigma\right)$ 33, Exercise 5.8.1.B].

Suppose now that a small $\delta>0$ (say, $\delta \leq \frac{1}{2}\left\|q_{0}\right\|_{\infty}$ ) is given and let $a_{0}$ be such that the conclusions of Theorem 1.1 and Corollary 3.2 are satisfied. For $a \in \mathbb{N}$, $a \geq a_{0}$, set

$$
Y:=\left\{q \in L^{\infty}\left(\mathbb{R}, \mathbb{R}^{N}\right): q(t)=\sum_{i \in \mathbb{Z}} s_{i} q_{0}(t-a i)+v(t), s_{i} \in\{0,1\},\|v\|_{\infty} \leq \delta\right\} .
$$

So here we have $\theta_{i}=a i, I_{i}=\left(a\left(i-\frac{1}{2}\right), a\left(i+\frac{1}{2}\right)\right)$, and $\left(\left\{s_{j}\right\}_{j \in \mathbb{Z}}, v\right) \mapsto q$ is a bijection between $\Sigma_{2} \times\left\{\|v\|_{\infty} \leq \delta\right\}$ and $Y$. In $Y$ we introduce a metric $d$ by setting

$$
d(q, 0)=\sum_{i \in \mathbb{Z}} 2^{-|i|}\left(s_{i}\left\|q_{0}\right\|_{\infty}+\|v\|_{L^{\infty}\left(I_{i}, \mathbb{R}^{N}\right)}\right) .
$$

Since $q_{0}$ decays exponentially and $\left\|q_{0}\right\|_{\infty}=\left\|q_{0}\right\|_{L^{\infty}\left(I_{0}, \mathbb{R}^{N}\right)}=\left\|q_{0}(\cdot-a i)\right\|_{L^{\infty}\left(I_{i}, \mathbb{R}^{N}\right)}$, it is easy to verify that the topology induced by $d$ and the $L_{l o c}^{\infty}\left(\mathbb{R}, \mathbb{R}^{N}\right)$-topology coincide on $Y$. Let

$$
X:=\{q \in Y: q \text { is a solution of (1.1) }\} .
$$

We shall show that $X$ is compact. If $\left(q^{m}\right)$ is a sequence in $X$, then $\left(s^{m}\right)=\left(s_{i}^{m}\right)_{i \in \mathbb{Z}}$ has a convergent subsequence because $\Sigma_{2}$ is compact. The argument of Corollary 3.3 shows that $v^{m} \rightarrow v$ in $L_{l o c}^{\infty}\left(\mathbb{R}, \mathbb{R}^{N}\right)$ after passing to a subsequence, $\|v\|_{\infty} \leq \delta$ and the corresponding function $q$ is in $X$.

Let $T_{a}: X \rightarrow X$ be the mapping given by $\left(T_{a} q\right)(t):=q(t+a)$, let $f_{a}: \mathbb{R}^{2 N} \rightarrow \mathbb{R}^{2 N}$ be the Poincaré (or time- $a$ ) mapping, $f_{a}(\hat{p}, \hat{q}):=(\dot{q}(a), q(a))$, where $q=q(t)$ is the solution of (1.1) satisfying the initial conditions $q(0)=\hat{q}, \dot{q}(0)=\hat{p}$, and $E v$ : $X \rightarrow \mathbb{R}^{2 N}$ the evaluation mapping, $E v(q):=(\dot{q}(0), q(0))$. Since $X$ is compact and $E v$ continuous and injective, it is a homeomorphism between $X$ and $I:=E v(X)$. Moreover, the diagram

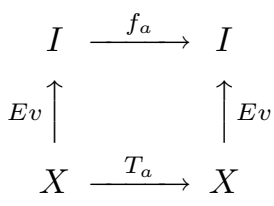


is commutative. Note that a priori $f_{a}$ maps $I$ into $\mathbb{R}^{2 N}$; however, $f_{a}(I) \subset I$ by the commutativity of (4.4).

Theorem 4.1. Under the assumptions of Theorem 1.1, if $\delta$ is small and $a \in \mathbb{N}$ large enough, then $\left(I, f_{a}\right)$ is semiconjugate to $\left(\Sigma_{2}, \sigma\right)$. Moreover, to each periodic sequence $s \in \Sigma_{2}$ there correspond a periodic point $(\hat{p}, \hat{q}) \in g^{-1}(s)$ for $f_{a}$, with the same minimal period as $s$, and a periodic solution $\bar{q} \in X$ of (1.1) such that $\operatorname{Ev}(\bar{q})=(\hat{p}, \hat{q})$.

Proof. Let $\bar{q}=\sum_{i \in \mathbb{Z}} s_{i} q_{0}(t-a i)+v(t) \in X$ and $\varphi(\bar{q}):=s=\left(s_{i}\right)_{i \in \mathbb{Z}}$. Then $\varphi: X \rightarrow \Sigma_{2}$ is continuous, according to Corollary 3.3 it is surjective, and it is easy to verify that the diagram

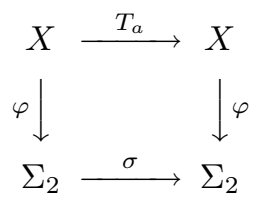

is commutative. Hence setting $g:=\varphi \circ(E v)^{-1}$ and using (4.4), (4.5) we see that also the diagram (4.3), with $M=I$ and $f=f_{a}$, is commutative. Hence the first conclusion.

Let $s=\left(s_{i}\right)_{i \in \mathbb{Z}}$ be a periodic sequence having minimal period $m$. By Corollary 3.2 there exists an $a m$-periodic solution $\bar{q}=\sum_{i \in \mathbb{Z}} s_{i} q_{0}(t-a i)+v(t) \in X$, it is clear that $\varphi(\bar{q})=s$, am is the minimal period of $\bar{q}$ and $E v(\bar{q})$ is a periodic point with minimal period $m$.

If the homoclinic $q_{0}$ in Theorem 1.1 is nondegenerate (in the sense that $N(L)=$ $\{0\})$, then $\left(I, f_{a}\right)$ is conjugate to $\left(\Sigma_{2}, \sigma\right)$; see 4 .

Remark 4.2. (i) As we have noted earlier, $h\left(f_{a}\right) \geq h(\sigma)=\log 2$; hence $h\left(f_{1}\right) \geq$ $(\log 2) / a$ according to [33, Theorem 5.8.4].

(ii) The conclusion about the entropy was obtained by Séré [30] for first order Hamiltonian systems with convex Hamiltonian. He has also shown that there exists an injective mapping from $\Sigma_{2}$ to $I$ which is continuous up to $\delta$ (the same conclusion follows from our arguments as well) and that the inverse of this mapping is uniformly continuous (which implies semiconjugacy). However, existence of periodic solutions has not been considered in [30].

Let

$$
\Sigma_{m+1}:=\{0,1, \ldots, m\}^{\mathbb{Z}}
$$

be the set of doubly infinite sequences of $m+1$ symbols with the metric (4.1), where $\left|s_{i}-\tilde{s}_{i}\right|=1$ unless $s_{i}=\tilde{s}_{i}$. The mapping $\sigma: \Sigma_{m+1} \rightarrow \Sigma_{m+1}$ given by (4.2) will be called the Bernoulli shift on $m+1$ symbols. By [33, Example 5.8.4] again, $h(\sigma)=\log (m+1)$.

Suppose $q_{1}, \ldots, q_{m}$ are isolated homoclinics as in Corollary 3.1 and let $a \geq a_{0}$ be such that the conclusions of Corollary 3.1 and of Corollary [3.2 for the case of $m$ homoclinics, are valid. Let

$\tilde{Y}:=\left\{q \in L^{\infty}\left(\mathbb{R}, \mathbb{R}^{N}\right): q(t)=\sum_{i \in \mathbb{Z}} q_{s_{i}}(t-a i)+v(t), s_{i} \in\{0,1, \ldots, m\},\|v\|_{\infty} \leq \delta\right\}$,

where here (and only here) we set $q_{0}(t):=0$, and let

$$
\widetilde{X}:=\{q \in \widetilde{Y}: q \text { is a solution of (1.1) }\} .
$$


Defining $f_{a}, T_{a}$ as before and setting $\widetilde{I}:=E v(\widetilde{X})$, we have the following extension of Theorem 4.1 .

Corollary 4.3. Under the assumptions of Corollary 3.1, if $\delta$ is small and $a \in \mathbb{N}$ large enough, then $\left(\widetilde{I}, f_{a}\right)$ is semiconjugate to $\left(\Sigma_{m+1}, \sigma\right)$. Moreover, to each periodic sequence $s \in \Sigma_{m+1}$ there correspond a periodic point $(\hat{p}, \hat{q}) \in g^{-1}(s)$ for $f_{a}$, with the same minimal period as $s$, and a periodic solution $\bar{q} \in \widetilde{X}$ of (1.1) such that $\operatorname{Ev}(\bar{q})=(\hat{p}, \hat{q})$.

In view of the comments following Corollary 3.1 we can interpret the above corollary as follows. Suppose $W$ is even in $q$ and $q_{0}$ is as in Theorem 1.1. Let $\delta$ be small and $a \in \mathbb{N}$ appropriately large. For each $i \in \mathbb{Z}$ we can choose (at random, say) $s_{i} \in\{0,1,2\}$, and this choice will decide whether a solution $\bar{q} \in \widetilde{X}$ will shadow $0, q_{0}$ or $-q_{0}$ in the interval $I_{i}$, i.e., whether $\|\bar{q}\|_{\infty},\left\|\bar{q}-q_{0}\right\|_{\infty}$ or $\left\|\bar{q}+q_{0}\right\|_{\infty}$ will be less than $2 \delta$ in $I_{i}$. Suppose now $q_{j}$ is a $j$-bump solution obtained with the aid of Theorem 1.1 $j=1, \ldots, m$. Then choosing $s_{i} \in\{0,1, \ldots, m\}$ will decide whether a solution $\bar{q} \in \widetilde{X}$ will shadow $0, q_{1}, \ldots$ or $q_{m}$ in $I_{i}$ (and thus have $0,1, \ldots$ or $m$ bumps in this interval).

\section{HOMOCLINICS WITH NONTRIVIAL CRITICAL GROUP}

It is easy to see that $\left(H_{1}\right)-\left(H_{3}\right)$ and (1.3) imply $\Phi(q)=\frac{1}{2}\|q\|^{2}+o\left(\|q\|^{2}\right)$ and $\Phi^{\prime}(q)=q+o(\|q\|)$ as $q \rightarrow 0$ (see e.g. [13]). Hence, denoting the open ball and the sphere of radius $\rho$ and center at $q$ respectively by $B_{\rho}(q)$ and $S_{\rho}(q)$, we have the following

Lemma 5.1. There exist $\alpha, \rho>0$ such that $\Phi(q)>0$ and $\left\langle\Phi^{\prime}(q), q\right\rangle>0$ for all $q \in B_{\rho}(0) \backslash\{0\}$. Moreover, $\Phi(q) \geq \alpha$ for all $q \in S_{\rho}(0)$.

Suppose $\Phi^{0} \backslash\{0\} \neq \emptyset$, and let

$$
\Gamma:=\left\{\gamma \in C([0,1], E): \gamma(0)=0, \gamma(1) \in \Phi^{0} \backslash\{0\}\right\}
$$

and

$$
c:=\inf _{\gamma \in \Gamma} \max _{s \in[0,1]} \Phi(\gamma(s)) .
$$

Clearly, $c \geq \alpha$ according to Lemma [5.1. In order to obtain homoclinics with nontrivial critical groups we need to introduce further assumptions:

$\left(H_{4}\right) \quad 0<\mu W(q, t) \leq q \cdot W_{q}(q, t)$ for some $\mu>2$ and all $q \neq 0, t \in \mathbb{R}$;

$\left(H_{5}\right) W(q, t)=\frac{1}{2} B_{\infty}(t) q \cdot q+\widetilde{W}(q, t)$, where $\widetilde{W}_{q}(q, t)=o(|q|)$ uniformly in $t$ as $|q| \rightarrow \infty$ and $B_{\infty}$ is an $N \times N$ symmetric matrix with 1-periodic entries;

$\left(H_{6}\right)$ there exists $q^{*} \in \mathbb{R}^{N}$ such that $\left(B(t)-B_{\infty}(t)\right) q^{*} \cdot q^{*}<0$ for all $t \in \mathbb{R}$;

$\left(H_{7}\right) q \cdot W_{q}(q, t)<W_{q q}(q, t) q \cdot q$ for all $q \neq 0, t \in \mathbb{R}$.

$\left(H_{4}\right)$ is the usual Ambrosetti-Rabinowitz superlinearity condition, while $\left(H_{5}\right)$ is an asymptotic linearity condition for $W_{q} .\left(H_{6}\right)$ implies that $\Phi$ has the mountain pass geometry if $\left(H_{1}\right)-\left(H_{3}\right)$ and $\left(H_{5}\right)$ hold. Finally, $\left(H_{7}\right)$ implies (as will be shown) that the Nehari manifold indeed is a manifold.

A simple example of $W$ satisfying $\left(H_{2}\right)-\left(H_{4}\right)$ and $\left(H_{7}\right)$ is $W(q, t)=\frac{1}{s}|q|^{s}, s>2$. Now let $h=h(s)$ be a function of class $C^{1}$ for $s \geq 0$ and suppose $h(0)=0$, $h^{\prime}(s)>0$ for all $s>0$ and $\lim _{s \rightarrow \infty} h(s)=h_{\infty}$. Set $H(s):=\int_{0}^{s} \sigma h(\sigma) d \sigma$ and $W(q, t):=H(|q|)$. Then $W$ satisfies $\left(H_{2}\right),\left(H_{3}\right),\left(H_{5}\right)$ with $B_{\infty}=h_{\infty} I,\left(H_{6}\right)$ if $h_{\infty}$ is large enough, and $\left(H_{7}\right)$. 
As we have already seen, if $q_{0}$ is a homoclinic, so is $q_{0}(\cdot+a), a \in \mathbb{Z}$. We shall call two homoclinics $q_{0}$ and $q_{1}$ geometrically distinct if $q_{1} \neq q_{0}(\cdot+a)$ for any $a \in \mathbb{Z}$.

Theorem 5.2. Suppose $B$ and $W$ satisfy $\left(H_{1}\right)-\left(H_{4}\right)$. If there exists $\varepsilon>0$ such that $\Phi^{c+\varepsilon}$ (where $c$ is as in (5.1)), contains only finitely many geometrically distinct critical points, then $C_{1}\left(\Phi, q_{0}\right) \neq 0$ for some $q_{0} \in K(c)$.

Proof. By [13, Theorem 2.19] and our assumption, $K(c)$ is nonempty and consists of isolated points. Using [13, Proposition 2.22] we can find a finite set $A=$ $\left\{q_{1}, \ldots, q_{k}\right\} \subset K(c)$ with the property that given $\bar{\varepsilon}_{1}, r_{1}$ small enough, there exist $\varepsilon_{1} \in\left(0, \bar{\varepsilon}_{1}\right)$ and $\gamma \in \Gamma$ such that $\Phi(\gamma(s)) \leq c+\varepsilon_{1}$ for all $s \in[0,1]$ and $\gamma(s) \in \bar{B}_{r_{1}}\left(q_{1}\right) \cup \cdots \cup \bar{B}_{r_{1}}\left(q_{k}\right)$ whenever $\Phi(\gamma(s)) \geq c-\varepsilon_{1}$. Moreover, choosing $\bar{\varepsilon}_{1}, r_{1}$ smaller if necessary, each $\bar{B}_{r_{1}}\left(q_{j}\right)$ is contained in a Gromoll-Meyer pair $\left(W_{j}, W_{j}^{-}\right)$ for $\Phi$ and $q_{j}$, and by Lemma 2.2. $\Phi$ satisfies the Palais-Smale condition in $W_{j}$. Hence it follows from the deformation lemma [26, Lemma 8.3] that we may assume $\Phi(\gamma(s)) \leq c$ for all $s$. Let $\gamma_{j}$ be the portion of $\gamma$ contained in $\bar{B}_{r_{1}}\left(q_{j}\right)$. For at least one $j, \gamma_{j} \subset \Phi^{c} \cap \bar{B}_{r_{1}}\left(q_{j}\right)$ and $\gamma_{j} \not \subset \Phi^{c} \cap \bar{B}_{r_{1}}\left(q_{j}\right) \backslash\left\{q_{j}\right\}$ (otherwise one could construct $\gamma$ with $\max _{s \in[0,1]} \Phi(\gamma(s))<c$, contradicting the definition of $c$ ). Now it is easy to see that $C_{1}\left(\Phi, q_{j}\right) \neq 0$ (cf. the argument of Theorem 8.7 in [26]).

Theorems 1.1 and 5.2 imply the existence of $k$-bump solutions, with $a$ independent of $k$. Also the results of Sections 3 and 4 apply here. Hence we have recovered (and slightly extended) the main results of [13, 15].

Next we show that if $\left(H_{7}\right)$ is satisfied, then homoclinics having nontrivial critical groups can be obtained by constrained minimization. Let

$$
\mathcal{M}:=\left\{q \in E:\left\langle\Phi^{\prime}(q), q\right\rangle=0, q \neq 0\right\}
$$

be the Nehari manifold.

Lemma 5.3. Assume $\left(H_{1}\right)-\left(H_{3}\right),\left(H_{7}\right)$ and either $\left(H_{4}\right)$ or $\left(H_{5}\right),\left(H_{6}\right)$. Then $\mathcal{M} \neq \emptyset$. Moreover, $\mathcal{M}$ is a closed $C^{1}$-submanifold of codimension 1 in $E$.

Proof. If $\left(H_{4}\right)$ is satisfied, then $W(q, t) \geq c_{1}|q|^{\mu}-c_{2}$ for some $c_{1}, c_{2}>0$ and all $q$. Let $q_{0} \in E, q_{0} \neq 0$. Then

$$
f(s):=\left\langle\Phi^{\prime}\left(s q_{0}\right), s q_{0}\right\rangle=s^{2}\left\|q_{0}\right\|^{2}-\int_{\mathbb{R}} s q_{0} \cdot W_{q}\left(s q_{0}, t\right) d t,
$$

and it is easily seen that $f(s)>0$ for small $s>0$ while $f(s)<0$ for $s$ large. Hence $f\left(s_{0}\right)=0$ and $s_{0} q_{0} \in \mathcal{M}$ for some $s_{0}>0$. So $\mathcal{M} \neq \emptyset$.

If $\left(H_{5}\right)$ and $\left(H_{6}\right)$ hold, $\left(B(t)-B_{\infty}(t)\right) q^{*} \cdot q^{*}<-\varepsilon_{0}\left|q^{*}\right|^{2}$ for all $t \in \mathbb{R}$ and some $q^{*} \in \mathbb{R}^{N}, \varepsilon_{0}>0$. Let $q_{0}(t)=\varphi(t) q^{*}$, where $\varphi \in C_{0}^{\infty}(\mathbb{R})$ is such that $\left\|q_{0}\right\|_{2}=1$ and $\left\|\dot{q}_{0}\right\|_{2}^{2} \leq \varepsilon<\varepsilon_{0}$. Also in this case $f(s)>0$ for small $s>0$ according to (5.2). Furthermore,

$$
\begin{aligned}
f(s) & =s^{2} \int_{\mathbb{R}}\left(\left|\dot{q}_{0}\right|^{2}+\left(B(t)-B_{\infty}(t)\right) q_{0} \cdot q_{0}\right) d t-\int_{\mathbb{R}} s q_{0} \cdot \widetilde{W}_{q}\left(s q_{0}, t\right) d t \\
& \leq s^{2}\left(\varepsilon-\varepsilon_{0}\right)+o\left(s^{2}\right) \quad \text { as } s \rightarrow \infty
\end{aligned}
$$

(that the integral containing $\widetilde{W}_{q}$ is $o\left(s^{2}\right)$ follows from the dominated convergence theorem). Hence $f(s)<0$ for large $s$, so $\mathcal{M} \neq \emptyset$ again.

Since 0 is an isolated point in the set $\left\{q \in E:\left\langle\Phi^{\prime}(q), q\right\rangle=0\right\}$ in view of Lemma 5.1. $\mathcal{M}$ is closed. Let $J(q):=\left\langle\Phi^{\prime}(q), q\right\rangle$. Then $J \in C^{1}(E, \mathbb{R}),\left.J\right|_{\mathcal{M}}=0$ and if 
$q \in \mathcal{M}$

$$
\left\langle J^{\prime}(q), q\right\rangle=\left\langle J^{\prime}(q), q\right\rangle-2 J(q)=\int_{\mathbb{R}}\left(q \cdot W_{q}(q, t)-W_{q q}(q, t) q \cdot q\right) d t<0
$$

according to $\left(H_{7}\right)$. Hence $J^{\prime} \neq 0$ on $\mathcal{M}$ and $\mathcal{M}$ is a $C^{1}$-manifold of codimension 1.

Lemma 5.4. Under the assumptions of the preceding lemma, let $q \in \mathcal{M}$ and

$$
h(s):=\Phi(s q)=\frac{1}{2} s^{2}\|q\|^{2}-\int_{\mathbb{R}} W(s q, t) d t, \quad s \geq 0 .
$$

Then $h^{\prime}(s)>0$ for $0<s<1$ and $h^{\prime}(s)<0$ for $s>1$. In particular, $h(s)<h(1)$ for all $s \geq 0, s \neq 1$ and $\max _{s \geq 0} \Phi(s q)=\Phi(q)$.

Proof. Since $q \in \mathcal{M}$ and

$$
h^{\prime}(s)=s\|q\|^{2}-\int_{\mathbb{R}} q \cdot W_{q}(s q, t) d t,
$$

$h^{\prime}(1)=0$. It follows from $\left(H_{7}\right)$ that $s \mapsto q \cdot W_{q}(s q, t) / s, s>0$, is strictly increasing. Hence the conclusion.

Theorem 5.5. Assume $\left(H_{1}\right)-\left(H_{3}\right),\left(H_{7}\right)$ and either $\left(H_{4}\right)$ or $\left(H_{5}\right)-\left(H_{6}\right)$. Let $c:=$ $\inf _{q \in \mathcal{M}} \Phi(q)$. Then $c$ is attained and $c>0$. Moreover, $q_{0} \in \mathcal{M}$ is a critical point of $\Phi$ if $\Phi\left(q_{0}\right)=c$, and $C_{1}\left(\Phi, q_{0}\right) \neq 0$ if $q_{0}$ is an isolated minimizer on $\mathcal{M}$.

It is interesting to compare Theorem 5.2 and the part of Theorem 5.5 which deals with superquadratic $W$. In both cases the hypotheses $\left(H_{1}\right)-\left(H_{4}\right)$ are needed. In order to obtain the conclusion concerning the critical group $C_{1}$ in Theorem 5.2. it was necessary to assume $\Phi^{c+\varepsilon}$ contains only finitely many geometrically distinct critical points. In Theorem 5.5 this condition was replaced by the weaker one that there is an isolated critical point at the level $c$. However, this was done at the expense of introducing the more restrictive hypothesis $\left(H_{7}\right)$.

In view of the results contained in [17 and 34 we expect that there is a result similar to Theorem 5.2 also in the asymptotically linear case, with $\left(H_{7}\right)$ replaced by some weaker conditions such as in [17, 34.

Proof of Theorem [5.5. Since $h(1)>h(0)=0$, where $h(s)$ is as in Lemma 5.4. $\Phi(q)>0$ for all $q \in \mathcal{M}$. So if $c$ is attained, $c>0$.

Let $\left(q_{m}\right) \subset \mathcal{M}, \Phi\left(q_{m}\right) \rightarrow c$. By Ekeland's variational principle (see e.g. [26]) we can choose $q_{m}$ so that $\left(\left.\Phi\right|_{\mathcal{M}}\right)^{\prime}\left(q_{m}\right) \rightarrow 0$. Since $\left\langle\Phi^{\prime}\left(q_{m}\right), q_{m}\right\rangle=0$ and $\left\langle J^{\prime}\left(q_{m}\right), q_{m}\right\rangle<$ 0 according to (5.3) (so $q_{m}$ is transversal to $\mathcal{M}$ ), it follows that $\Phi^{\prime}\left(q_{m}\right) \rightarrow 0$ and $\left(q_{m}\right)$ is a Palais-Smale sequence.

We need to show that $\left(q_{m}\right)$ is bounded. Since this is well known in the superlinear case, see e.g. [13, it suffices to consider asymptotically linear $W_{q}$. We adapt an argument which may be found in 20. Suppose $\left\|q_{m}\right\| \rightarrow \infty$ and set $w_{m}:=$ $\alpha q_{m} /\left\|q_{m}\right\|$, where $\alpha^{2}>2 c$. If

$$
\sup _{t \in \mathbb{R}} \int_{t-r}^{t+r}\left|w_{m}(t)\right|^{2} d t \rightarrow 0 \quad \text { as } m \rightarrow \infty
$$

for some $r>0$, then $w_{m} \rightarrow 0$ in $L^{s}\left(\mathbb{R}, \mathbb{R}^{N}\right)$ for all $s \in(2, \infty)$ according to P.L. Lions' vanishing lemma [36, Lemma 1.21]. In view of $\left(H_{3}\right)$ and $\left(H_{5}\right)$, for each $\varepsilon>0$ there 
exists $C_{\varepsilon}$ such that $|W(q, t)| \leq \varepsilon|q|^{2}+C_{\varepsilon}|q|^{s}\left(s>2\right.$ fixed). Hence $\int_{\mathbb{R}} W\left(w_{m}, t\right) d t \rightarrow$ 0 and

$$
\Phi\left(w_{m}\right)=\frac{1}{2}\left\|w_{m}\right\|^{2}-\int_{\mathbb{R}} W\left(w_{m}, t\right) d t \rightarrow \alpha^{2} / 2 .
$$

By Lemma 5.4. $\Phi\left(w_{m}\right) \leq \Phi\left(q_{m}\right) \rightarrow c$ and therefore $\alpha^{2} / 2 \leq c<\alpha^{2} / 2$, a contradiction. So vanishing cannot occur and hence there exist $t_{m}$ such that

$$
\lim _{m \rightarrow \infty} \int_{t_{m}-r}^{t_{m}+r}\left|w_{m}(t)\right|^{2} d t \geq \eta>0
$$

for almost all $m$. Taking a larger $r$ we may assume $t_{m} \in \mathbb{Z}$; hence setting $\widetilde{w}_{m}(t):=$ $w_{m}\left(t_{m}+t\right)$ we obtain

$$
\int_{-r}^{r}\left|\widetilde{w}_{m}(t)\right|^{2} d t \geq \eta
$$

Since $\left\|\widetilde{w}_{m}\right\|=\left\|w_{m}\right\|=\alpha, \widetilde{w}_{m} \rightarrow \widetilde{w}$ in $E$ and $\widetilde{w}_{m} \rightarrow \widetilde{w}$ in $L_{\text {loc }}^{2}\left(\mathbb{R}, \mathbb{R}^{N}\right)$ and a.e. in $\mathbb{R}$ after passing to a subsequence. It follows from (5.5) that $\widetilde{w} \neq 0$. Let $\widetilde{q}_{m}(t):=$ $q_{m}\left(t_{m}+t\right)$. Then $\left(\widetilde{q}_{m}\right)$ is a Palais-Smale sequence by the periodicity of $B$ and $W$. Hence for each $v \in E$,

$$
\int_{\mathbb{R}}\left(\dot{\widetilde{q}}_{m} \cdot \dot{v}+\left(B(t)-B_{\infty}(t)\right) \widetilde{q}_{m} \cdot v\right) d t-\int_{\mathbb{R}} \widetilde{W}_{q}\left(\widetilde{q}_{m}, t\right) \cdot v d t \rightarrow 0
$$

and therefore

$$
\int_{\mathbb{R}}\left(\dot{\widetilde{w}}_{m} \cdot \dot{v}+\left(B(t)-B_{\infty}(t)\right) \widetilde{w}_{m} \cdot v\right) d t-\alpha \int_{\mathbb{R}} \widetilde{W}_{q}\left(\widetilde{q}_{m}, t\right) \cdot v /\left\|\widetilde{q}_{m}\right\| d t \rightarrow 0 .
$$

Letting $m \rightarrow \infty$ and using the dominated convergence theorem in the second integral above we see that

$$
-\ddot{\widetilde{w}}+\left(B(t)-B_{\infty}(t)\right) \widetilde{w}=0 .
$$

However, since $B$ and $B_{\infty}$ are 1-periodic, $\widetilde{w}$ must be 0 as it follows from [18, Proposition 2.2]. This contradiction shows $\left(q_{m}\right)$ is bounded.

For the sequence $\left(q_{m}\right)$ which is bounded by now, we use a similar argument again. If (5.4) is satisfied, then $q_{m} \rightarrow 0$ in $L^{s}\left(\mathbb{R}, \mathbb{R}^{N}\right)$, so

$$
\left\|q_{m}\right\|^{2}=\int_{\mathbb{R}} q_{m} \cdot W_{q}\left(q_{m}, t\right) d t \rightarrow 0
$$

and $0 \in \mathcal{M}$, a contradiction. Therefore (5.5) holds with $\widetilde{w}_{m}$ replaced by $\widetilde{q}_{m}$, so passing to a subsequence, $\widetilde{q}_{m} \rightarrow q_{0} \neq 0$ in $E, \widetilde{q}_{m} \rightarrow q_{0}$ a.e. in $\mathbb{R}, \Phi^{\prime}\left(\widetilde{q}_{m}\right) \rightarrow$ 0 and $\Phi\left(\widetilde{q}_{m}\right) \rightarrow c$. But then $\Phi^{\prime}\left(q_{0}\right)=0$ because $\left\langle\Phi^{\prime}\left(q_{m}\right), v\right\rangle \rightarrow\left\langle\Phi^{\prime}\left(q_{0}\right), v\right\rangle$ for all $v \in E$ as is easy to see. We shall show that $c$ is attained at $q_{0}$. By $\left(H_{7}\right)$, $\frac{d}{d s}\left(\frac{1}{2} W_{q}(s q, t) \cdot q-W(s q, t)\right)>0$ for all $q \neq 0$ and $s>0$. Therefore $\frac{1}{2} W_{q}(q, t) \cdot q-$ $W(q, t) \geq 0$, and it follows from Fatou's lemma that

$$
\begin{aligned}
c+o(1) & =\Phi\left(\widetilde{q}_{m}\right)-\frac{1}{2}\left\langle\Phi^{\prime}\left(\widetilde{q}_{m}\right), \widetilde{q}_{m}\right\rangle=\int_{\mathbb{R}}\left(\frac{1}{2} W_{q}\left(\widetilde{q}_{m}, t\right) \cdot \widetilde{q}_{m}-W\left(\widetilde{q}_{m}, t\right)\right) d t \\
& \geq \int_{\mathbb{R}}\left(\frac{1}{2} W_{q}\left(q_{0}, t\right) \cdot q_{0}-W\left(q_{0}, t\right)\right) d t+o(1) \\
& =\Phi\left(q_{0}\right)-\frac{1}{2}\left\langle\Phi^{\prime}\left(q_{0}\right), q_{0}\right\rangle+o(1)=\Phi\left(q_{0}\right)+o(1) .
\end{aligned}
$$

So $c=\inf _{\mathcal{M}} \Phi \leq \Phi\left(q_{0}\right) \leq c$. 
It is clear that each $q_{0}$ minimizing $\Phi$ on $\mathcal{M}$ is a critical point. Suppose $q_{0}$ is isolated and let $\gamma$ be the line segment $s q_{0}, 1-\varepsilon \leq s \leq 1+\varepsilon, \varepsilon>0$ small. Then $\gamma \subset \Phi^{c} \cap \bar{B}_{2 \varepsilon}\left(q_{0}\right)$ and $\gamma \not \subset \Phi^{c} \cap \bar{B}_{2 \varepsilon}\left(q_{0}\right) \backslash\left\{q_{0}\right\}$ according to Lemma 5.4 hence $C_{1}\left(\Phi, q_{0}\right) \neq 0$.

Now consider (1.1) with $B(t)=B_{0}+\varepsilon B_{1}(t)$, where $\varepsilon>0, B_{0}$ is a positive definite constant matrix and $W=W(q)$. This is a perturbation of the autonomous system

$$
-\ddot{q}+B_{0} q=W^{\prime}(q),
$$

and the corresponding functional is

$$
\Phi_{\varepsilon}(q)=\frac{1}{2} \int_{\mathbb{R}}\left(|\dot{q}|^{2}+B_{0} q \cdot q\right) d t+\frac{1}{2} \varepsilon \int_{\mathbb{R}} B_{1}(t) q \cdot q d t-\int_{\mathbb{R}} W(q) d t .
$$

We shall denote the Nehari manifold for this problem by $\mathcal{M}_{\varepsilon}$, and we write $\Phi_{0}, \mathcal{M}_{0}$ if $\varepsilon=0$. Although it is possible to allow more general time-periodic perturbations of the system (5.6), for simplicity we prefer to restrict ourselves to the situation described above. Note that these more general perturbations have been considered in [7, however, under the assumption that (5.6) has a solution $q_{0}$ with $N\left(\Phi_{0}^{\prime \prime}\left(q_{0}\right)\right)=$ $\mathbb{R} \dot{q}_{0}$. In [7] the relation to the Melnikov function is also discussed.

Theorem 5.6. Assume $\left(H_{1}\right)-\left(H_{3}\right),\left(H_{7}\right)$ and either $\left(H_{4}\right)$ or $\left(H_{5}\right)-\left(H_{6}\right)$ for small $\varepsilon>0$, where $B$ and $W$ are as above. Let $c_{\varepsilon}:=\inf _{q \in \mathcal{M}_{\varepsilon}} \Phi_{\varepsilon}(q)$ and let $\delta \in\left(0, c_{0} / 2\right)$. If $\varepsilon>0$ is small enough and $\Phi_{\varepsilon}^{c_{\varepsilon}+\delta}$ has only finitely many geometrically distinct critical points, then there exists a critical point $\tilde{q}$ for $\Phi_{\varepsilon}$ such that $\Phi_{\varepsilon}(\tilde{q}) \in$ $\left(c_{\varepsilon}, c_{\varepsilon}+\delta\right) \subset\left(c_{\varepsilon}, 2 c_{\varepsilon}\right)$ and $C_{2}\left(\Phi_{\varepsilon}, \tilde{q}\right) \neq 0$.

Proof. We claim that for a fixed $R>0$, if $q \in \mathcal{M}_{0} \cap \bar{B}_{R}(0)$ and $s q \in \mathcal{M}_{\varepsilon}$, then $s \rightarrow 1$ uniformly in $q$ as $\varepsilon \rightarrow 0$. Let

$$
h_{\varepsilon}(s):=\Phi_{\varepsilon}(s q)=\frac{1}{2} s^{2} \int_{\mathbb{R}}\left(|\dot{q}|^{2}+B_{0} q \cdot q\right) d t+\frac{1}{2} s^{2} \varepsilon \int_{\mathbb{R}} B_{1}(t) q \cdot q d t-\int_{\mathbb{R}} W(s q) d t .
$$

Then by Lemma 5.4. $h_{0}^{\prime}$ is positive for $0<s<1$ and negative for $s>1$. Hence for each $\varepsilon_{0}>0, h_{0}^{\prime}\left(1-\varepsilon_{0}\right)>0$ and therefore

$$
h_{\varepsilon}^{\prime}\left(1-\varepsilon_{0}\right)=h_{0}^{\prime}\left(1-\varepsilon_{0}\right)+\left(1-\varepsilon_{0}\right) \varepsilon \int_{\mathbb{R}} B_{1}(t) q \cdot q d t>0
$$

for all $q \in \mathcal{M}_{0} \cap \bar{B}_{R}(0)$ provided $\varepsilon$ is small enough. Similarly, $h_{\varepsilon}^{\prime}\left(1+\varepsilon_{0}\right)<0$, so $s q \in \mathcal{M}_{\varepsilon}$ for some $s \in\left(1-\varepsilon_{0}, 1+\varepsilon_{0}\right)$, and the claim follows.

Given a small $\varepsilon>0$, there exists a minimizer $q_{0}$ for $\Phi_{\varepsilon}$ on $\mathcal{M}_{\varepsilon}$, and it follows from the claim above that $\Phi_{\varepsilon}\left(q_{0}\right)=c_{\varepsilon}>\delta$. Let $q_{\tau}(t):=q_{0}(t+\tau)$. Then $q_{1}$ is also a minimizer by the $\mathbb{Z}$-invariance of $\Phi_{\varepsilon}$, and $q_{0}, q_{1}$ are isolated critical points according to our assumptions. Choose $\sigma$ so that $\sigma q_{0} \in \mathcal{M}_{0}$. Then $\sigma q_{\tau} \in \mathcal{M}_{0}$ for all $\tau$ by the $\mathbb{R}$-invariance of $\Phi_{0}$, and there exists $s=s(\tau) \in C([0,1], \mathbb{R})$ such that $s(\tau) q_{\tau} \in \mathcal{M}_{\varepsilon}$. It follows from the claim that for any $\varepsilon_{0}>0$ and $0 \leq \tau \leq 1, s(\tau) \in\left(1-\varepsilon_{0}, 1+\varepsilon_{0}\right)$ whenever $\varepsilon$ is sufficiently small (note that $s(0)=s(1)=1$ as $q_{0}, q_{1} \in \mathcal{M}_{\varepsilon}$ ). Set

$$
\widetilde{\Gamma}:=\left\{\gamma \in C\left([0,1], \mathcal{M}_{\varepsilon}\right): \gamma(0)=q_{0}, \gamma(1)=q_{1}\right\}
$$

and

$$
\tilde{c}:=\inf _{\gamma \in \widetilde{\Gamma}} \max _{\tau \in[0,1]} \Phi(\gamma(\tau))
$$


Since $q_{0}$ and $q_{1}$ are isolated, $\tilde{c}>\Phi_{\varepsilon}\left(q_{0}\right)=c_{\varepsilon}$. As

$$
\Phi_{\varepsilon}(q)-\Phi_{0}(q)=\frac{1}{2} \varepsilon \int_{\mathbb{R}} B_{1}(t) q \cdot q d t,
$$

it is easy to see that for all $\varepsilon$ small enough,

$$
\Phi_{0}\left(\sigma q_{\tau}\right)<c_{\varepsilon}+\delta / 2 \quad \text { and } \quad\left|\Phi_{\varepsilon}\left(s(\tau) q_{\tau}\right)-\Phi_{0}\left(\sigma q_{\tau}\right)\right|<\delta / 2, \quad 0 \leq \tau \leq 1 .
$$

Hence $\max _{\tau \in[0,1]} \Phi_{\varepsilon}\left(s(\tau) q_{\tau}\right)<c_{\varepsilon}+\delta$ and $c_{\varepsilon}<\tilde{c}<c_{\varepsilon}+\delta<2 c_{\varepsilon}$ because $\tau \mapsto$ $s(\tau) q_{\tau} \in \widetilde{\Gamma}$.

We remark here that since $q \in \mathcal{M}_{\varepsilon}$ is transversal to the tangent space to $\mathcal{M}_{\varepsilon}$ at $q,\left(q_{m}\right) \subset \mathcal{M}_{\varepsilon}$ is a Palais-Smale sequence for $\left.\Phi_{\varepsilon}\right|_{\mathcal{M}_{\varepsilon}}$ if and only if it is a Palais-Smale sequence for $\Phi_{\varepsilon}$ in $E$. Therefore [13, Proposition 1.24] applies to $\left.\Phi_{\varepsilon}\right|_{\mathcal{M}_{\varepsilon}}$. Keeping this in mind, an inspection shows that the arguments of [13, Propositions 2.3 and 2.22] go through for deformations on $\mathcal{M}_{\varepsilon}$. Hence we see as in the proof of Theorem 5.2 that there exists a critical point $\tilde{q} \in \Phi_{\varepsilon}^{-1}(\tilde{c})$ such that $C_{1}\left(\left.\Phi_{\varepsilon}\right|_{\mathcal{M}_{\varepsilon}}, \tilde{q}\right) \neq 0$. If $\left(\widetilde{W}, \widetilde{W}^{-}\right)$is a Gromoll-Meyer pair and $\widetilde{V}$ a corresponding pseudogradient field for $\left.\Phi_{\varepsilon}\right|_{\mathcal{M}_{\varepsilon}}$ and $\tilde{q}$, it is easy to see that $\left(W, W^{-}\right):=h\left(\left(\widetilde{W}, \widetilde{W}^{-}\right) \times([-1,1],\{-1,1\})\right)$, where $h(q, s)=(1+\rho s) q\left(\rho>0\right.$ small enough) is a Gromoll-Meyer pair for $\Phi_{\varepsilon}$ and $\tilde{q}$ in $E$ (a corresponding vector field is e.g. $V((1+\rho s) q)=\widetilde{V}(q)-\rho s q)$. Hence

$$
H_{*}\left(W, W^{-}\right)=H_{*}\left(\widetilde{W}, \widetilde{W}^{-}\right) \otimes H_{*}([-1,1],\{-1,1\})=H_{*+1}\left(\widetilde{W}, \widetilde{W}^{-}\right)
$$

and $C_{2}\left(\Phi_{\varepsilon}, \tilde{q}\right) \neq 0$.

Note that if $\tilde{c}>2 c_{\varepsilon}$, then there are multibumps close to the level $2 c_{\varepsilon}$ and the finiteness assumption of Theorem [5.6 will never be satisfied below the level $\tilde{c}$. Therefore it is important to have $c_{\varepsilon}+\delta<2 c_{\varepsilon}$.

We point out that by Theorems 1.1 and 5.6 we obtain new multibump solutions whose existence does not follow from Theorems 5.2 and 5.5 .

\section{SChröDinger EQUATION}

In this final section we consider the semilinear Schrödinger equation

$$
-\Delta u+V(x) u=g(x, u), \quad x \in \mathbb{R}^{N}, u \in \mathbb{R} .
$$

Denote the spectrum of $-\Delta+V$ in $L^{2}\left(\mathbb{R}^{N}\right)$ by $\sigma(-\Delta+V)$, set $G(x, u):=\int_{0}^{u} g(x, s) d s$, $2^{*}:=2 N /(N-2)$ if $N \geq 3$ and suppose the following hypotheses are satisfied:

$\left(S_{1}\right) V \in C\left(\mathbb{R}^{N}, \mathbb{R}\right)$ is 1 -periodic in each of the variables $x_{1}, \ldots, x_{N}$ and $\sigma(-\Delta+V) \subset(0, \infty)$

$\left(S_{2}\right) g, g_{u} \in C\left(\mathbb{R}^{N} \times \mathbb{R}, \mathbb{R}\right)$ are 1-periodic in $x_{1}, \ldots, x_{N}$;

$\left(S_{3}\right)\left|g_{u}(x, u)-g_{u}(x, v)\right| \leq C(1+|u|+|v|)^{2^{*}-2-\alpha}|u-v|^{\alpha}$ for all $u, v \in \mathbb{R}$ and some $C>0, \alpha \in\left(0, \min \left\{1,2^{*}-2\right\}\right)$ if $N \geq 3$,

$\left|g_{u}(x, u)-g_{u}(x, v)\right| \leq C(1+|u|+|v|)^{p}|u-v|^{\alpha}$ for all $u, v \in \mathbb{R}$ and some $C>0, \alpha \in(0,1], p>0$ if $N=2$;

$\left(S_{4}\right) g(x, 0)=g_{u}(x, 0)=0$ for all $x \in \mathbb{R}^{N}$.

Note that in $\left(S_{1}\right)$ we have made no assumption that $V \geq 0$ (cf. Lemma 6.2 below). Hypothesis $\left(S_{3}\right)$ is a condition of local uniform Hölder (or Lipschitz if $\alpha=1$ ) continuity with a growth restriction on the Hölder (or Lipschitz) constant. A slightly different variant of $\left(S_{3}\right)$ may be found in [27, p. 277]. It is easy to verify that $g(x, u)=|u|^{p-2} u$ satisfies $\left(S_{3}\right)$ for any $p \in(2, \infty)$ if $N=2$ and any $p \in\left(2,2^{*}\right]$ if $N \geq 3$ (if $p<3$, then $\alpha=p-2$, otherwise $\alpha=1$ ). 
Let $E:=H^{1}\left(\mathbb{R}^{N}\right)$ and

$$
\left.\langle u, v\rangle:=\int_{\mathbb{R}^{N}}(\nabla u \cdot \nabla v)+V(x) u v\right) d x, \quad\|u\|:=\langle u, u\rangle^{1 / 2} .
$$

It follows from $\left(S_{1}\right)$ that $\langle.,$.$\rangle is an equivalent inner product in E$ (see e.g. [32]). The functional corresponding to (6.1) is

$$
\Phi(u)=\int_{\mathbb{R}^{N}}\left(|\nabla u|^{2}+V(x) u^{2}\right) d x-\int_{\mathbb{R}^{N}} G(x, u) d x \equiv \frac{1}{2}\|u\|^{2}-\int_{\mathbb{R}^{N}} G(x, u) d x,
$$

and it is well known that $\Phi \in C^{2}(E, \mathbb{R})$ and if $\Phi^{\prime}(u)=0$, then $u$ is a solution of (6.1). Moreover, $|u(x)| \leq C e^{-\lambda|x|}$ for all $x \in \mathbb{R}^{N}$ and some $C, \lambda>0$. To see this, we first note that $u \in L^{\infty}\left(\mathbb{R}^{N}\right)$ and $u(x) \rightarrow 0$ as $|x| \rightarrow \infty$ (see e.g. [9]). Now we follow the argument of [27, Theorem 6.1] (where $g$ of subcritical growth has been considered) and re-write (6.1) as

$$
-\Delta u+\widetilde{V}(x) u \equiv-\Delta u+(V(x)-g(x, u) / u) u=0 .
$$

Since $u$ is continuous (by standard elliptic estimates) and tends to 0 as $|x| \rightarrow \infty$, $\widetilde{V} \in L^{\infty}\left(\mathbb{R}^{N}\right)$ and $\widetilde{V}(x)-V(x) \rightarrow 0$ as $|x| \rightarrow \infty$. Therefore $\sigma_{\text {ess }}(-\Delta+\widetilde{V})=$ $\sigma_{\text {ess }}(-\Delta+V) \subset(0, \infty)$ and as $0 \in \sigma(-\Delta+\widetilde{V})$, the conclusion follows from 31, Theorem C.3.5].

Let $\theta=\left(\xi_{1}, \ldots, \xi_{N}\right) \in \mathbb{R}^{N}$,

$$
(\theta * u)(x):=u(x-\theta)
$$

and

$$
|\theta-\widetilde{\theta}|_{\infty}:=\max _{1 \leq j \leq N}\left|\xi_{j}-\widetilde{\xi}_{j}\right| \quad\left(\text { the maximum norm in } \mathbb{R}^{N}\right)
$$

The following result is an analogue of Theorem 1.1 for the Schrödinger equation:

Theorem 6.1. Suppose the hypotheses $\left(S_{1}\right)-\left(S_{4}\right)$ are satisfied and $u_{0} \in E \backslash\{0\}$ is an isolated (in $E$ ) solution of (6.1) such that $C_{*}\left(\Phi, u_{0}\right) \neq 0$. For each $\delta>0$ there exists $a \in \mathbb{N}$ with the property that if $k \geq 2, \theta_{1}, \theta_{2}, \ldots, \theta_{k} \in \mathbb{Z}^{N}$ and $\left|\theta_{i}-\theta_{j}\right|_{\infty} \geq 3 a$ for all $i \neq j$, then there is $v \in E$ such that $\|v\|_{\theta} \leq \delta$ (the $\theta$-norm will be defined below) and $\bar{u}=\theta_{1} * u_{0}+\ldots+\theta_{k} * u_{0}+v$ is a solution of (6.1).

The proof is similar to that of Theorem 1.1, therefore we only point out the main differences. Let

$$
\Theta_{k}^{a}:=\left\{\theta=\left(\theta_{1}, \ldots, \theta_{k}\right) \in \mathbb{Z}^{N k}:\left|\theta_{i}-\theta_{j}\right|_{\infty} \geq 3 a \text { if } i \neq j\right\},
$$

and for each $\theta \in \Theta_{k}^{a}$ let $Q_{1}, \ldots, Q_{l}(l \geq k)$ be open sets such that $\mathbb{R}^{N}=\bigcup_{i=1}^{l} \bar{Q}_{i}$, $Q_{i} \cap Q_{j}=\emptyset$ if $i \neq j$, each $\widetilde{Q}_{j}:=\left\{x \in \mathbb{R}^{N}: \operatorname{dist}\left(x, Q_{j}\right)<a / 4\right\}$ intersects at most $4^{N} Q_{i}$ 's, and for $1 \leq i \leq k,\left\{x:\left|x-\theta_{i}\right|_{\infty}<a / 2\right\} \subset Q_{i}$. It is easy to see that such a decomposition of $\mathbb{R}^{N}$ exists and moreover, one can choose all bounded sets $Q_{i}$ to be cubes with edges parallel with the coordinate axes and of length $\geq a$. Let

$$
\|u\|_{\theta}:=\max _{1 \leq i \leq l}\|u\|_{i}, \text { where }\|u\|_{i}^{2}:=\int_{Q_{i}}\left(|\nabla u|^{2}+V(x) u^{2}\right) d x .
$$

The norms $\|\cdot\|$ and $\|\cdot\|_{\theta}$ are equivalent for each fixed $\theta$ and $\|\cdot\|_{\theta} \leq\|\cdot\|$. In Lemma 2.4 it is easy to see that $\left|\nabla \chi_{j}(x)\right| \leq c / a$ for a suitable constant $c$. Since $\widetilde{Q}_{j}$ intersects at most $4^{N} Q_{i}$ 's, this gives the conclusion. As in the proof of Lemma [2.5. set $\left\|w_{m}\right\|_{j_{m}}=\left\|w_{m}\right\|_{\theta^{m}}$. Passing to a subsequence, either for all $m$ we have $\theta_{j_{m}}^{m} \in Q_{j_{m}}^{m}$, or for all $m$ there is no $i \in\left\{1, \ldots, k_{m}\right\}$ such that $\theta_{i}^{m} \in Q_{j_{m}}^{m}$. In the 
first case we may assume employing the $\mathbb{Z}^{N}$-invariance of $\Phi$ that $\theta_{j_{m}}^{m}=0$ for all $\mathrm{m}$ and continuing as in Lemma 2.5 we obtain

$$
\liminf _{m \rightarrow \infty} \int_{\mathbb{R}^{N}} g_{u}\left(x, u_{0}^{a_{m}}+u_{m}\right) w_{m} v_{m} d x \geq \frac{1}{2},
$$

where $u_{0}(x) \rightarrow 0$ as $|x| \rightarrow \infty$, and since $\left\|u_{m}\right\|_{\theta^{m}}=\left\|u_{m}\right\|_{j_{m}} \rightarrow 0, u_{m} \rightarrow 0$ in $H^{1}\left(\widetilde{Q}_{j_{m}}\right)$. Hence by $\left(S_{3}\right)$ and $\left(S_{4}\right)$ (assuming $N \geq 3$; the case $N=2$ is similar),

$$
\begin{aligned}
& \left|\int_{\mathbb{R}^{N} \backslash Q} g_{u}\left(x, u_{0}^{a_{m}}+u_{m}\right) w_{m} v_{m} d x\right| \\
& \quad \leq C \int_{\widetilde{Q}_{j_{m} \backslash Q}}\left(\left|u_{0}^{a_{m}}+u_{m}\right|^{\alpha}+\left|u_{0}^{a_{m}}+u_{m}\right|^{2^{*}-2}\right)\left|w_{m}\right|\left|v_{m}\right| d x \\
& \quad \leq \varepsilon\left\|w_{m}\right\|_{j_{m}}^{2}=\varepsilon
\end{aligned}
$$

for a suitable cube $Q$ and $m$ large enough (cf. (2.12) ). Since $u_{0}^{a_{m}}+u_{m} \rightarrow u_{0}$ and $w_{m} \rightarrow 0$ in $H^{1}(Q)$,

$$
\int_{Q} g_{u}\left(x, u_{0}^{a_{m}}+u_{m}\right) w_{m} v_{m} d x \rightarrow 0,
$$

which gives the desired contradiction. In the second case we may assume $0 \in Q_{j_{m}}^{m}$ and the argument becomes simpler: $u_{0}^{a_{m}}$ is replaced by 0 and it is easy to see that $\int_{\mathbb{R}^{N}} g_{u}\left(x, u_{m}\right) w_{m} v_{m} d x \rightarrow 0$ while $\left\langle w_{m}, \chi_{m} w_{m}\right\rangle$ does not. In Lemma 2.7 it follows from $\left(S_{3}\right)$ and the inclusion $\operatorname{supp} z_{j}^{a} \subset Q_{j}$ that

$$
\begin{aligned}
\int_{\mathbb{R}^{N}} \mid g_{u}\left(x, u_{0}^{a}+u\right) & -g_{u}\left(x, u_{0}\right)|| w|| z_{j}^{a} \mid d x \\
& \leq C_{1} \int_{\mathbb{R}^{N}}\left(1+|u|+\left|u_{0}\right|\right)^{2^{*}-2-\alpha}\left(|u|^{\alpha}+\left|u_{0}-u_{0}^{a}\right|^{\alpha}\right)|w|\left|z_{j}^{a}\right| d x
\end{aligned}
$$

In Lemma 2.8 we now have

$$
\begin{aligned}
\left|\left\langle\left(\Phi^{\prime \prime}(u)-\Phi^{\prime \prime}(\widetilde{u})\right) w, v\right\rangle\right| & \leq \int_{\mathbb{R}^{N}}\left|g_{u}(x, u)-g_{u}(x, \widetilde{u})\right||w||v| d x \\
& \leq C \int_{\mathbb{R}^{N}}(1+|u|+|\widetilde{u}|)^{2^{*}-2-\alpha}|u-\widetilde{u}|^{\alpha}|w||v| d x
\end{aligned}
$$

Since $\|u-\widetilde{u}\|_{j} \leq\|u-\widetilde{u}\|_{\theta} \leq \delta$, the conclusion follows using the Sobolev embedding theorem and the argument of this lemma. The remaining parts of the proof of Theorem 6.1 follow the corresponding ones in Section 2 and require mainly notational changes.

It is clear that Corollary 3.1 has its counterpart here and so does Corollary 3.2 if one decomposes $\mathbb{R}^{N}$ in a different way. Let $Q^{k}$ be a cube having vertices in $\mathbb{Z}^{N}$, edges of length $k a$ ( $a$ even), and subdivide it into $k^{N}$ equal cubes $Q_{i}^{k}$. Then one can work in the space $H_{p e r}^{1}\left(Q^{k}\right)$ of functions $u \in H_{l o c}^{1}\left(\mathbb{R}^{N}\right)$ which are $k a$-periodic in each variable and, for $a$ large enough and independent of $k$, find periodic $k^{N}$-bump solutions of (6.1).

Similarly one obtains $m$-bump solutions of (6.1) in $E$ if one chooses $m$ cubes $Q_{1}, \ldots, Q_{m}$ from $\left\{Q_{i}^{k}\right\}$, lets $\theta_{1}, \ldots, \theta_{m}$ be their centers and finds $Q_{m+1}, \ldots, Q_{l}$ as above. Since $\|v\|_{\theta} \leq \delta$, using standard elliptic estimates we see that the solution $\bar{u}$, and hence also $v$, is uniformly bounded in $L^{\infty}\left(\mathbb{R}^{N}\right)$. This leads to solutions of the 
form

$$
\bar{u}(x)=\sum_{i \in \mathbb{Z}^{N}} s_{i} u_{0}(x-a i)+v(x), \quad s_{i} \in\{0,1\},\|v\|_{\theta} \leq \delta .
$$

Set $\Sigma:=\{0,1\}^{\mathbb{Z}^{N}}$ and define (in the terminology of [4]) the multishift operator $\sigma^{m} \in C(\Sigma, \Sigma)$ by setting $\left(\sigma^{m}(s)\right)_{j}:=s_{j+m}, j, m \in \mathbb{Z}^{N}$. Further, let $X$ be the set of solutions of the form (6.2) $,\left(T_{a}^{m} u\right)(x):=u(x+a m)$ and $\varphi(\bar{u})=s=\left(s_{i}\right)_{i \in \mathbb{Z}^{N}}$, $\bar{u} \in X$. Then $\varphi(X)=\Sigma$ and the diagram

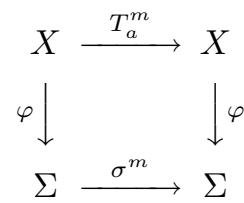

is commutative. Moreover, $\varphi^{-1}(s)$ contains a periodic solution for each $s$ which is periodic in all variables. This result corresponds to Theorem 4.1. If $N(L)=\{0\}$, then $\varphi$ is invertible according to [4. Note that for our Newtonian systems all multishifts $\sigma^{m}$ are generated by $\sigma$, while for Schrödinger equations the $N$ "elementary" shifts $\sigma^{e_{j}}, 1 \leq j \leq N$, are needed in order to generate all $\sigma^{m}\left(e_{j}\right.$ is the $j$-th element of the standard basis in $\mathbb{R}^{N}$ ).

It is not difficult to adapt Theorems [5.2, 5.5] and [5.6 to the present situation. According to Theorem 6.1 and the comments above, this would extend the main results of [14] and 34] (however, assumption $\left(S_{3}\right)$ is more restrictive than the growth restrictions there). Instead of working out the details, we focus on the equation

$$
-\Delta u+V(x) u=|u|^{2^{*}-2} u, \quad x \in \mathbb{R}^{N}, N \geq 4 .
$$

Then $g(x, u)=|u|^{2^{*}-2} u$ satisfies $\left(S_{3}\right)$, as we have already observed, and, obviously, it satisfies $\left(S_{2}\right),\left(S_{4}\right)$. We also assume $\left(S_{1}\right)$ holds. If $V(x)=\lambda \neq 0$, then it is known [6] that (6.3) has only the trivial solution $u=0$ in $E$. Here we shall require $V$ to be sign-changing. That such $V$ can satisfy $\left(S_{1}\right)$ follows from the result below. Let $Q_{0}:=[0,1]^{N}$.

Lemma 6.2. If $V$ is continuous, 1-periodic in $x_{1}, \ldots, x_{N}$ and $\int_{Q_{0}} V(x) d x>0$, then $\sigma(-\Delta+V) \subset(0, \infty)$.

Proof. According to [22, p. 161], the bottom of $\sigma(-\Delta+V)$ equals the infimum of $\lambda_{1}(p)$ over all $p \in 2 \pi Q_{0}$, where $\lambda_{1}(p)$ is the first eigenvalue of $(i \nabla-p)^{2}$ in $H_{\text {per }}^{1}\left(Q_{0}, \mathbb{C}\right)$. Since

$$
\lambda_{1}(p)=\inf _{u \in H_{p e r}^{1}\left(Q_{0}, \mathbb{C}\right) \backslash\{0\}} \frac{\int_{Q_{0}}|(\nabla+i p) u|^{2} d x+\int_{Q_{0}} V(x)|u|^{2} d x}{\int_{Q_{0}}|u|^{2} d x},
$$

it is easy to see, e.g. by expanding $u$ in a Fourier series, that $\inf _{p \in Q_{0}} \lambda_{1}(p)>0$.

Let $\mathcal{M}$ denote the Nehari manifold again.

Theorem 6.3. Suppose $V$ satisfies $\left(S_{1}\right), V\left(x_{0}\right)<0$ for some $x_{0}$ and let $\Phi$ be the functional corresponding to (6.3). Then $c:=\inf _{u \in \mathcal{M}} \Phi(u)$ is attained and $c>0$. Moreover, $u_{0} \in \mathcal{M}$ is a critical point of $\Phi$ if $\Phi\left(u_{0}\right)=c$, and $C_{1}\left(\Phi, u_{0}\right) \neq 0$ if $u_{0}$ is an isolated minimizer on $\mathcal{M}$. 
Proof. Since $g(x, u) u<g_{u}(x, u) u^{2}$ for all $u \neq 0$, where $g(x, u)=|u|^{2^{*}-2} u, \mathcal{M}$ has the properties stated in Lemma 5.3. Also the conclusion of Lemma 5.4 holds here, therefore $c$ is the mountain pass level defined at the beginning of Section 5. By the argument of Theorem 5.5, if $u_{0} \in \mathcal{M}$ and $\Phi\left(u_{0}\right)=c$, then $u_{0}$ is a critical point of $\Phi$ and $C_{1}\left(\Phi, u_{0}\right) \neq 0$ provided $u_{0}$ is an isolated minimizer.

It remains to show that $c$ is attained. This, however, follows implicitly from 8, as will be explained below. A special case of the result contained there asserts that if $0 \notin \sigma(-\Delta+V)$ and $\sigma(-\Delta+V) \cap(-\infty, 0) \neq \emptyset$, then (6.3) has a nontrivial solution if $N \geq 4$. If $\sigma(-\Delta+V) \subset(0, \infty)$ and $V\left(x_{0}\right)<0$ for some $x_{0}$, then the argument in [8] still applies and becomes in fact simpler. Our functional here has the mountain pass instead of the linking geometry, and we obtain a sequence $\left(u_{m}\right)$ such that $\Phi^{\prime}\left(u_{m}\right) \rightarrow 0$ and $\Phi\left(u_{m}\right) \rightarrow c$. This sequence is bounded, and by the argument of [8] it cannot be vanishing, because $c<S^{N / 2} / N$ as $V\left(x_{0}\right)<0$, where

$$
S:=\inf _{u \in E \backslash\{0\}} \frac{\|\nabla u\|_{2}^{2}}{\|u\|_{2^{*}}^{2}} .
$$

Therefore, possibly after translating $u_{m}$ by some $x_{m} \in \mathbb{Z}^{N}, u_{m} \rightarrow u_{0} \neq 0$ and $\Phi^{\prime}\left(u_{0}\right)=0$. Since

$$
\Phi\left(u_{m}\right)=\Phi\left(u_{m}\right)-\frac{1}{2}\left\langle\Phi^{\prime}\left(u_{m}\right), u_{m}\right\rangle+o(1)=\frac{1}{N}\left\|u_{m}\right\|_{2^{*}}^{2^{*}}+o(1) \rightarrow c
$$

and $\Phi\left(u_{0}\right)=\frac{1}{N}\left\|u_{0}\right\|_{2^{*}}^{2^{*}}, \Phi\left(u_{0}\right) \leq c$. Since there are no solutions $u \neq 0$ below the level $c$, we must have $\Phi\left(u_{0}\right)=c$.

Corollary 6.4. Suppose $V$ satisfies $\left(S_{1}\right), V\left(x_{0}\right)<0$ for some $x_{0}$ and $\Phi$ is the functional corresponding to (6.3). If there exists an isolated minimizer for $\Phi$ on $\mathcal{M}$, then (6.3) possesses multibump solutions described in Theorem 6.1. Moreover, since $\Phi$ is even, there also exist multibumps of the form $\bar{u}=s_{1} \theta_{1} * u_{0}+\cdots+s_{k} \theta_{k} * u_{0}+v$, where $s_{i}=1$ or $-1,1 \leq i \leq k$.

\section{ACKNOWLEDGEMENT}

The authors would like to thank Yanyan Li for sending the thesis [37, and the anonymous referee for carefully reading the manuscript and for helpful comments.

\section{REFERENCES}

[1] N. Ackermann, A nonlinear superposition principle and multibump solutions of periodic Schrödinger equations, J. Funct. Anal. 234 (2006), 277-320. MR2216902 (2007b:47182)

[2] N. Ackermann and T. Weith, Multibump solutions of nonlinear periodic Schrödinger equations in a degenerate setting, Comm. Contemp. Math. 7 (2005), 269-298. MR2151860 (2006f:35069)

[3] S. Alama and Y.Y. Li, On "multibump" bound states for certain semilinear elliptic equations, Indiana Univ. Math. J. 41 (1992), 983-1026. MR.1206338 (94d:35044)

[4] S. Angenent, The shadowing lemma for elliptic PDE. In: Dynamics of infinite-dimensional systems, S.N. Chow and J.K. Hale eds., Springer-Verlag, Berlin, 1987, pp. 7-22. MR.921893 (89b:58165)

[5] G. Arioli, F. Gazzola and S. Terracini, Multibump periodic motions of an infinite lattice of particles, Math. Z. 223 (1996), 627-642. MR1421960 (98d:58150)

[6] V. Benci and G. Cerami, Existence of positive solutions of the equation $-\Delta u+a(x) u=$ $u^{(N+2) /(N-2)}$ in $\mathbb{R}^{N}$, J. Func. Anal. 88 (1990), 90-117. MR.1033915 (91f:35097)

[7] M. Berti and P. Bolle, Homoclinics and chaotic behaviour for perturbed second order systems, Ann. Mat. Pura Appl. (4) 176 (1999), 323-378. MR.1746547(2001c:37065) 
[8] J. Chabrowski and A. Szulkin, On a semilinear Schrödinger equation with critical Sobolev exponent, Proc. Amer. Math. Soc. 130 (2002), 85-93. MR1855624 (2002i:35053)

[9] J. Chabrowski and A. Szulkin, On the Schrödinger equation involving a critical exponent and magnetic field, Topol. Meth. in Nonl. Anal. 25 (2005), 3-21. MR.2133390 (2005k:35110)

[10] K.C. Chang, Infinite Dimensional Morse Theory and Multiple Solution Problem, Birkhäuser, Boston, 1993. MR 1196690(94e:58023)

[11] W.A. Coppel, Dichotomies in Stability Theory, Lecture Notes in Mathematics 629, SpringerVerlag, Berlin, 1978. MR0481196 (58:1332)

[12] V. Coti Zelati, I. Ekeland and E. Séré, A variational approach to homoclinic orbits in Hamiltonian systems, Math. Ann. 288 (1990), 133-160. MR.1070929 (91g:58065)

[13] V. Coti Zelati and P.H. Rabinowitz, Homoclinic orbits for second order Hamiltonian systems possessing superquadratic potentials, J. Amer. Math. Soc. 4 (1991), 693-727. MR1119200 (93e:58023)

[14] V. Coti Zelati and P.H. Rabinowitz, Homoclinic type solutions for a semilinear elliptic PDE on $R^{n}$, Comm. Pure Appl. Math. 45 (1992), 1217-1269. MR.1181725 (93k:35087)

[15] V. Coti Zelati and P.H. Rabinowitz, Multibump periodic solutions of a family of Hamiltonian systems, Topol. Meth. Nonl. Anal. 4 (1994), 31-57. MR1321808(96a:58042)

[16] E.N. Dancer, Degenerate critical points, homotopy indices and Morse inequalities, J. Reine Angew. Math. 350 (1984), 1-22. MR743531 (85i:58033)

[17] Y. Ding, Multiple homoclinics in a Hamiltonian system with asymptotically or super linear terms, Comm. Contemp. Math. 8 (2006), 453-480. MR2258874 (2007h:37092)

[18] Y. Ding and M. Willem, Homoclinic orbits of a Hamiltonian system, Z. Angew. Math. Phys. 50 (1999), 759-778. MR.1721793 (2000k:37086)

[19] A. Dold, Lectures on Algebraic Topology, Springer-Verlag, Berlin, 1972. MR0415602 (54:3685)

[20] L. Jeanjean, On the existence of bounded Palais-Smale sequences and application to a Landesman-Lazer type problem set on $\mathbf{R}^{N}$, Proc. Roy. Soc. Edinb. 129A (1999), 787-809. MR:1718530 (2001c:35034)

[21] W. Kryszewski and A. Szulkin, An infinite dimensional Morse theory with applications, Trans. Amer. Math. Soc. 349 (1987), 3181-3234. MR1422612 (97m:58035)

[22] P. Kuchment, Floquet Theory for Partial Differential Equations, Birkhäuser, Basel, 1993. MR.1232660 (94h:35002)

[23] Y.Y. Li and Z.Q. Wang, Gluing approximate solutions of minimum type on the Nehari manifold, Electron. J. Diff. Eq., Conference 06, 2001, 215-223. MR1804776 (2001m:58027)

[24] Z.L. Liu and Z.Q. Wang, Multi-bump type nodal solutions having a prescribed number of nodal domains, I and II, Ann. IHP, Analyse Non Linéaire 22 (2005), pp. 597-608 and 609631. MR2171994 (2006f:35094)

[25] R. Magnus, The implicit function theorem and multi-bump solutions of periodic partial differential equations, Proc. Roy. Soc. Edinburgh 136 (2006), 559-583. MR2227808(2007k:47116)

[26] J. Mawhin and M. Willem, Critical Point Theory and Hamiltonian Systems, Springer-Verlag, New York, 1989. MR982267 (90e:58016)

[27] A. Pankov, Periodic nonlinear Schrödinger equation with application to photonic crystals, Milan J. Math. 73 (2005), 259-287. MR2175045 (2006h:35087)

[28] P.H. Rabinowitz, Variational methods for Hamiltonian systems. In: Handbook of Dynamical Systems, Vol. 1A, B. Hasselblatt and A. Katok eds., North-Holland, Amsterdam, 2002, pp. 1091-1127. MR1928531(2004a:37081)

[29] E. Séré, Existence of infinitely many homoclinic orbits in Hamiltonian systems, Math. Z. 209 (1992), 27-42. MR1143210 (92k:58201)

[30] E. Séré, Looking for the Bernoulli shift, Ann. IHP, Analyse Non Linéaire 10 (1993), 561-590. MR1249107 (95b:58031)

[31] B. Simon, Schrödinger semigroups, Bull. Amer. Math. Soc. 7 (1982), 447-526. MR670130 (86b:81001a)

[32] C.A. Stuart, Bifurcation into spectral gaps, Bull. Belg. Math. Soc., Supplement, 1995. MR1361485 (96m:47115)

[33] W. Szlenk, An Introduction to the Theory of Smooth Dynamical Systems, Polish Scientific Publishers, Warsaw and Wiley, New York, 1984. MR791919 (86f:58042)

[34] F.A. van Heerden, Homoclinic solutions for a semilinear elliptic equation with an asymptotically linear nonlinearity, Calc. Var. PDE 20 (2004), 431-455. MR2071929 (2005c:35098) 
[35] S. Wiggins, Global Bifurcations and Chaos, Springer-Verlag, New York, 1988. MR.956468 (89m:58057)

[36] M. Willem, Minimax Theorems, Birkhäuser, Boston, 1996. MR1400007(97h:58037)

[37] H.Y. Xu, Critical exponent elliptic equations: gluing and the moving sphere method, Thesis, Rutgers, 2007.

Dipartimento di Matematica, Politecnico di Milano, Piazza L. Da Vinci 32, 20133 Milano, Italy

E-mail address: gianni.arioli@polimi.it

Department of Mathematics, Stockholm University, 10691 Stockholm, Sweden

E-mail address: andrzejs@math.su.se

Department of Mathematical Sciences, Tsinghua University, Beijing 100084, People's Republic of China

E-mail address: wzou@math.tsinghua.edu.cn 